\title{
Quantum Toroidal Algebras and Their Vertex Representations
}

\author{
By
}

\author{
Yoshihisa SAITo*
}

\begin{abstract}
We construct the vertex representations of the quantum toroidal algebras $U_{q}\left(\mathfrak{G l}_{n+1, t o 1}\right)$. In the classical case the vertex representations are not irreducible. However in the quantum case they are irreducible.
\end{abstract}

\section{§1. Introduction}

The classical toroidal algebras have been studied by many authors [MEY]. $[\mathrm{S}],[\mathrm{Y}]$, etc. Here "classical" means $q=1$. The definition of the quantum toroidal algebras is given in $[\mathrm{GKV}]$. They gave a geometric realization of the quantum toroidal algebras without any results on their representation theory. Recently Varagnolo and Vasserot [VV] proved Schur-type duality between representations of the quantum toroidal algebras and the double affine Hecke algebra introduced by Cherednik $[\mathrm{C}]$. This is an analogue of the duality between the quantum affine algebras and the affine Hecke algebras given by Chari and Pressly $[\mathrm{CP}]$. In $[\mathrm{VV}]$ only the representations of "trivial central charge" was studied. It is known that there are two subalgebras $U_{q}^{(1)}\left(\widehat{\mathfrak{B l}}_{n+1}\right)$ and $U_{q}^{(2)}\left(\widehat{\mathfrak{S} \mathfrak{l}_{n+1}}\right)$ of $U_{g}\left(\mathfrak{S l}_{n+1, \text { tor }}\right)$ such that there are surjective algebra homomorphisms $U_{q}\left(\widehat{\mathfrak{G l}}_{n+1}\right) \rightarrow U_{q}^{(\imath)}\left(\hat{\mathfrak{G}}_{n+1}\right)$ for $i=1$,2. In this paper we say that $M$ has a level $(0,0)$ instead of the trivial central charge. The first 0 means that $M$ has a level 0 as a $U_{q}^{(1)}\left(\hat{\mathfrak{G l}}_{n+1}\right)$-module and the second 0 means that $M$ has a level 0 as a $U_{q}^{(2)}\left(\hat{\mathfrak{G l}}_{n+1}\right)$-module. This notation is an analogue of level 0 representations of the affine quantum algebras.

In this paper we try to consider an analogue of "integrable representations"

Communicated by T. Miwa, June 12, 1997

1991 Mathematics Subject Classification (s): 17B37. 81R50

* Research Institute for Mathematical Sciences, Kyoto University, Japan.

Current Address: Department of Mathematics. University of Hiroshıma. Higashi-Hiroshıma, Hiroshima. 739-8526. Japan.

The author is supported by the JSPS Research Fellowships for Young Scientists 
in the toroidal case. Let us recall the integrability of quantum Kac-Moody modules. Let $U_{q}(\mathfrak{g})$ be a quantum Kac-Moody algebra and $V$ a $U_{q}(\mathfrak{g})$-module. We say $V$ is integrable if $V$ has a weight space decomposition and locally nilpotent actions of the Chevalley generators of $U_{q}(\mathrm{~g})$. Therefore the definition of "integrability" needs Chevalley-type generators. The toroidal algebras is defined through Drinfeld type of generators and its Chevalley-type generators are not known. Therefore we are not able to define the integrability at this moment. However, in the affine case, Frenkel-Jing [FJ] realized the integrable representations with level 1 by the vertex representations. Thus if there are "vertex representations" of quantum toroidal algebras, they must be interesting example of the integrable representations still not defined. In the $q=1$ case, vertex representations of the toroidal algebras have been already considered by Moody-Eswara Rao-Yokonuma [MEY]. In this paper we construct the $q$-analogue of the representations defined by them for $\mathfrak{g}=\mathfrak{g l}_{n+1}$ with level $(1,0)$ and (1.1). Therefore we give a new class of the representations of the quantum toroidal algebras. In the $q=1$ case, the Fock modules are not irreducible over the Heisenberg algebra and the vertex representations are not irreducible over the toroidal algebra. In the quantum case, it is not the case: the Fock modules are irreducible and also the vertex representations with level (1,0) are irreducible.

The algebra $L_{q}\left(\mathfrak{g}_{t o r}\right)$ has infinitely many generators satisfying infinitely many relations (See $\S 2$ ). It is preferable that $U_{q}\left(g_{t o r}\right)$ is written by finitely many generators with finitely many relations. According to [GKV] there are finitely many generators of $U_{q}\left(\mathfrak{S l}_{2, t o r}\right)$ but the relations among these generators are highly non-trivial. In this paper we give an explicit form of finitely many generators of $U_{q}\left(\mathfrak{S l}_{2, t o r}\right)$ and closed relations of them (See $\left.\$ 4\right)$. They coincide with the generators by Vasserot [V].

\section{§2. Definition of Quantum Toroidal Algebras}

2.1. Notations. Let $\mathfrak{g}$ be a complex semisimple Lie algebra of type $A_{n}$ and $\widehat{g}$ an affine Kac-Moody Lie algebra of type $A_{n}^{(1)}$. We denote their Cartan subalgebras by $\mathfrak{h}$ and $\widehat{\mathfrak{h}}$ respectively. We denote by $\bar{\alpha}_{1}, \ldots, \bar{\alpha}_{n}$ the simple roots of $\mathrm{g}$, by $\bar{h}_{1}, \ldots, \bar{h}_{n}$ the simple coroots of $\mathrm{g}$. by $\bar{\Lambda}_{1}, \ldots, \bar{\Lambda}_{n}$ the fundamental weights of $\mathrm{g}$, by $\alpha_{0}, \ldots, \alpha_{n}$ the simple roots of $\hat{\mathrm{g}}$. by $h_{0}, \ldots, h_{n}$ the simple coroots of $\hat{\mathrm{g}}$ and $\Lambda_{0}$, $\ldots, \Lambda_{n}$ the fundamental weights of $\widehat{\mathrm{g}}$. Let $\bar{Q}=\bigoplus_{i=1}^{n} \mathbb{R} \bar{\alpha}_{1}$ be the root lattice of $\mathrm{g}, \bar{P}=$ $\bigoplus_{i=1}^{n} \mathbb{R} \bar{\Lambda}_{2}$ the weight lattice of $\mathfrak{g}, Q=\bigoplus_{i=0}^{n} \mathbb{Z} \alpha_{i}$ the root lattice of $\widehat{\mathrm{g}}$ and $P=$ $\bigoplus_{i=0}^{n} \mathbb{Z} \Lambda_{i} \oplus \mathbb{Z} \delta$ the weight lattice of $\hat{\mathrm{g}}$. Here $\delta$ is the null root.

We denote the pairing of $\mathfrak{h}$ and $\mathfrak{h}^{*}$ (resp. $\widehat{\mathfrak{h}}$ and $\widehat{\mathfrak{h}}^{*}$ ) by $\langle$,$\rangle . The invariant$ bilinear form on $P$ is given by $\left(\alpha_{i} \mid \alpha_{j}\right)=-\delta_{i j-1}+2 \delta_{i},-\delta_{i j+1}$ and $(\delta \mid \delta)=0$. The projection form $P$ to $\bar{P}$ is given by $\bar{\Lambda}_{2}=\Lambda_{2}-\Lambda_{0}$ and $\bar{\delta}=0$. 
2.2. We will give the definition of the quantum toroidal algebra $U_{q}\left(\mathfrak{g}_{t o r}\right)$.

Definition 2.2.1. Let $M=\left(m_{\imath \jmath}\right)_{0 \leq i, j \leq n}$ be a skew-symmetric $(n+1) \times(n+$ 1 ) - matrix with integral coefficients and let $\kappa$ be an element of $\mathbb{Q}(q) * U_{q}\left(g_{t o r}\right)$ is an associated algebra over $\mathbf{Q}(q)$ with generators:

$$
E_{\imath, k}, F_{i, k}, H_{\imath, l}, K_{\imath}^{ \pm}, q^{ \pm \frac{1}{2} c}, q^{ \pm d_{1}}, q^{ \pm d 2},
$$

for $k \in \mathbb{Z}, l \in \mathbb{Z} \backslash\{0\}$ and $i=0,1, \ldots, n$.

We introduce $K_{t, k}^{ \pm}$as the Fourier components of the following generating functions:

$$
\begin{gathered}
K_{\imath}^{+}(z)=\sum_{k \geq 0} K_{i, k}^{+} z^{-k}=K_{i}^{+} \exp \left(\left(q-q^{-1}\right) \sum_{k \geq 1} H_{\imath, k} z^{-k}\right), \\
K_{l}^{-}(z)=\sum_{k \leq 0} K_{i, k}^{-} z^{-k}=K_{l}^{-} \exp \left(-\left(q-q^{-1}\right) \sum_{k \geq 1} H_{1,-k} z^{k}\right) .
\end{gathered}
$$

The defining relations of $U_{q}\left(\mathrm{~g}_{\mathrm{tor}}\right)$ are then written as follows: $q^{ \pm \frac{1}{2} c}$ are central, $K_{i}^{+} K_{i}^{-}=K_{\imath}^{-} K_{i}^{+}=1$.

$$
\left[K_{i}^{ \pm}, K_{j}^{ \pm}\right]=0 \text {, }
$$

$$
\left[K_{1}^{ \pm}, H_{,, l}\right]=0 \text {, }
$$

$$
\left[H_{\imath, k}, H_{j, l}\right]=\delta_{k+l, 0} \frac{1}{k}\left[k\left\langle h_{\imath}, \alpha_{\jmath}\right\rangle\right] \frac{q^{k c}-q^{-k c}}{q-q^{-1}} \kappa^{-k m_{\imath}},
$$

$$
\begin{gathered}
{\left[q^{ \pm d_{t}}, K_{\jmath}^{ \pm}\right]=0,} \\
q^{d_{1}} H_{\jmath, l} q^{-d_{1}}=q^{l} H_{\jmath, l}, \\
{\left[q^{ \pm d_{2}}, H_{, l}\right]=0,} \\
q^{d_{1}} E_{\jmath, k} q^{-d_{1}}=q^{k} E_{j, k}, \\
q^{d_{1}} F_{\jmath, k} q^{-d_{1}}=q^{k} F_{j, k}, \\
q^{d_{2}} E_{\jmath, k} q^{-d_{2}}=q^{\delta_{\jmath} 0} E_{\jmath, k}, \\
q^{d_{2}} F_{\jmath, k} q^{-d_{2}}=q^{-\delta \jmath_{0}} F_{\jmath, k}, \\
K_{\imath}^{+} E_{\jmath, k} K_{\imath}^{-}=q^{\left\langle h_{\imath}, \alpha_{\jmath}\right\rangle} E_{\jmath, k}, \\
K_{\imath}^{+} F_{\jmath_{, k}} K_{\imath}^{-}=q^{-\left\langle h_{\imath}, \alpha_{\jmath}\right\rangle} F_{j, k},
\end{gathered}
$$

$$
\left[H_{i, k}, E_{\jmath, l}\right]=\frac{1}{k}\left[k\left\langle h_{\imath}, \alpha_{\jmath}\right\rangle\right] q^{-\frac{1}{2}|k| c} \kappa^{-k m_{v} E_{j, k+l}},
$$




$$
\begin{aligned}
& {\left[H_{\imath, k}, F_{\jmath, l}\right]=-\frac{1}{k}\left[k\left\langle h_{\imath}, \alpha_{\jmath}\right\rangle\right] q^{\frac{1}{2}|k| c} \kappa^{-k m_{t} F_{\jmath, k+l}},}
\end{aligned}
$$

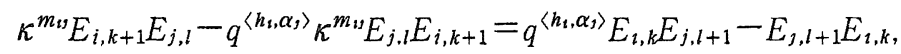

$$
\begin{aligned}
& \kappa^{m \imath v} F_{\imath, k+1} F_{j, l}-q^{\left.-\left\langle h_{1}, \alpha\right\rangle\right\rangle} \kappa^{m v} F_{j, l} F_{l, k+1}=q^{\left.-\left\langle h_{t}, \alpha\right\rangle\right\rangle} F_{i, k} F_{j, l+1}-F_{j, l+1} F_{\imath, k}, \\
& {\left[E_{\imath, k}, F_{j, l}\right]=\delta_{i, j} \frac{1}{q-q^{-1}}\left\{q^{\frac{1}{2}(k-l) c} K_{\imath, k+l}^{+}-q^{\frac{1}{2}(l-k) c} K_{\imath, k+l}^{-}\right\},}
\end{aligned}
$$

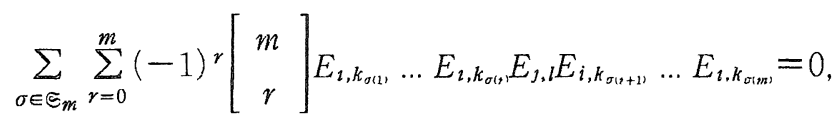

$$
\begin{aligned}
& \sum_{\sigma \in \Xi_{m}} \sum_{r=0}^{m}(-1)^{r}\left[\begin{array}{c}
r \\
m
\end{array}\right] F_{\imath, K_{\sigma(1)}} \ldots F_{l, k_{\sigma(1)}} F_{,, l} F_{i, k_{\sigma(r+1)}} \ldots F_{l, k_{\sigma(m)}}=0 .
\end{aligned}
$$

for $i \neq j$,

where $m=1-\left\langle h_{1}, \alpha_{j}\right\rangle$.

In these relations we denote $[k]=\frac{q^{k}-q^{-k}}{q-q^{-1}} .[n] !=\prod_{k=1}^{n}[k],\left[\begin{array}{c}m \\ r\end{array}\right]=$ $\frac{[m] !}{[r] ![m-r] !}$

2.3. Let $U_{q}^{\prime \prime}\left(\mathrm{g}_{t o r}\right)$ be the subalgebra of $U_{q}\left(\mathfrak{g}_{t o r}\right)$ generated by $E_{\imath, k}, F_{i, k}, K_{\imath}^{ \pm}, H_{\imath, l}$, $q^{ \pm \frac{1}{2} c}$. Let $L_{q}^{\phi^{\prime}}\left(\mathfrak{g}_{t o r}\right)$ (resp. $\left.U_{q}^{\prime \phi}\left(g_{t o r}\right)\right)$ be the subalgebra generated by $U_{q}^{\prime \prime}\left(g_{t o r}\right)$ and $q^{ \pm d_{1}}\left(\right.$ resp. $\left.q^{ \pm d_{2}}\right)$. Let $L_{q}^{(1)^{\prime}}\left(\hat{\mathfrak{S}}_{n+1}\right)$ be the subalgebra generated by $E_{\imath, k}, F_{i, k}, K_{\imath}^{ \pm}, H_{\imath, l}$, $q^{ \pm \frac{1}{2} c}\left(1 \leq_{i} \leq_{n}, k \in \mathbb{Z}, l \in \mathbb{Z} \backslash\{0\}\right)$ and $U_{q}^{(1)}\left(\widehat{\mathfrak{G l}}_{n+1}\right)$ the subalgebra generated by $U_{q}^{(1)^{\prime}}\left(\hat{\mathfrak{S}}_{n+1}\right)$ and $q^{ \pm d_{1}}$. Let $U_{q}^{(2)^{\prime}}\left(\hat{\mathfrak{G}}_{n+1}\right)$ the subalgebra generated by $E_{\imath, 0}, F_{i, 0}, K_{\imath}^{ \pm}(0$ $\left.\leq_{i} \leq n\right)$ and $U_{q}^{(2)}\left(\hat{\mathfrak{G}}_{n+1}\right)$ the subalgebra generated by $U_{q}^{(2)^{\prime}}\left(\hat{\mathfrak{S}}_{n+1}\right)$ and $q^{ \pm d_{2}}$. By the definition it is clear that there are surjective homomorphisms $U_{q}\left(\hat{\mathfrak{E}}_{n+1}\right) \rightarrow$ $U_{q}^{(2)}\left(\hat{\mathfrak{G l}}_{n+1}\right)$ and $U_{q}^{\prime}\left(\widehat{\mathfrak{G l}}_{n+1}\right) \rightarrow L_{q}^{r(2)^{\prime}}\left(\hat{\mathfrak{G}}_{n+1}\right)$.

The following are straightforward.

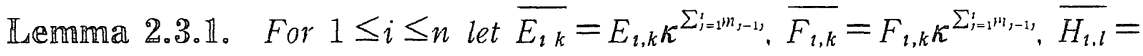
$H_{l, l} \kappa^{\Sigma_{t=1}^{t} m_{1-1}}$. Then the relations between $\overline{E_{l, k}} \overline{F_{l, k}}, \overline{H_{l, l}}$ and $K_{l}^{ \pm}$are precisely the relations of Drinfeld generators of $U_{q}\left(\hat{\mathfrak{S}}_{n+1}\right)$. That is, there are surjective homomorphisms $U_{q}\left(\widehat{\mathfrak{S l}}_{n+1}\right) \rightarrow L_{q}^{(1)}\left(\widehat{\mathfrak{S}}_{n+1}\right)$ and $\left.U_{q}^{\prime}\left(\hat{\mathfrak{S l}}_{n+1}\right) \rightarrow U_{q}^{(1)}\right)^{\prime}\left(\hat{\mathfrak{S l}}_{n+1}\right)$.

Lemma 2.3.2. Let $K_{\delta}^{ \pm}=\prod_{i=0}^{n} K_{i}^{ \pm}$. Then $K_{\delta}^{ \pm}$are central elements of $U_{q}\left(\mathfrak{g}_{t o r}\right)$.

Note that $q^{ \pm c}$ is the central elements of $U_{q}^{(1)}\left(\hat{\mathfrak{s l}}_{n+1}\right)$ and $K_{\delta}^{ \pm}$the central 
elements of $U_{q}^{(2)}\left(\hat{\mathfrak{G l}}_{n+1}\right)$.

\section{§3. Vertex Representations}

3.1. Heisenberg algebras. In this section we shall give the vertex representations of $U_{q}\left(\mathfrak{g}_{\text {tor }}\right)$. We assume $c=1$.

Consider a $\mathbf{Q}(q)$-algebra $S_{n}$ generated by $H_{i, l}(0 \leq i \leq n, l \in \mathbb{Z} \backslash\{0\})$ satisfying:

$$
\left[H_{i, k}, H_{,, l}\right]=\delta_{k+l, 0} \frac{1}{k}\left[k\left\langle h_{\imath}, \alpha_{\jmath}\right\rangle\right] \frac{q^{k}-q^{-k}}{q-q^{-1}} \kappa^{-k m_{\imath}} .
$$

We call $S_{n}$ the Heisenberg algebra.

Let $S_{n}^{+}\left(\right.$resp. $\left.S_{n}^{-}\right)$be the subalgebra of $S_{n}$ generated by $H_{i, l}\left(0 \leq_{i} \leq_{n, l}>0\right)$ (resp. $\left.0 \leq_{i} \leq n, l<0\right)$.

We introduce the Fock space

$$
\mathscr{F}_{n}=S_{n v^{\prime} 0}
$$

with the defining relations:

$$
H_{l, l i}{ }^{\prime}=0, \quad \text { for } l>0 \text {, }
$$

$$
q^{\frac{1}{2} c} v_{0}=q^{\frac{1}{2} v_{0}}
$$

Note that $\mathscr{F}_{n}$ is a free $S_{n}^{-}$-module of rank 1 .

Let $\mathbf{F}$ be a field of characteristic zero and let $a$ be an associative $\mathbf{F}$-algebra generated by $x_{p}, y_{p}\left(p \in \mathbb{Z}_{>0}\right), z$ and its inverse $z^{-1}$ with the following relations:

$$
\begin{gathered}
{\left[x_{p}, z\right]=\left[y_{p}, z\right]=0,} \\
{\left[x_{p}, x_{r}\right]=\left[y_{p}, y_{r}\right]=0,} \\
{\left[x_{p}, y_{r}\right]=\delta_{p r} z .}
\end{gathered}
$$

Let $\mathfrak{a}^{+}\left(\right.$resp. $\left.\mathfrak{a}^{-}\right)$be the subalgebra of $\mathfrak{a}$ generated by $x_{p}$ (resp. $\left.y_{p}\right)$. We set $\mathfrak{b}=\mathfrak{a}^{+}$ $\otimes \mathbf{F}\left[z, z^{-1}\right]$. This is a maximal abelian subalgebra of a. Fix a nonzero scalar $\lambda \in$ $\mathbb{F}^{\times}$. Let $\mathbf{F}_{\lambda}$ be the one-dimensional space $\mathbb{F}$ viewed as a $\mathfrak{b}$-module by:

$$
z \cdot 1=\lambda, \quad \mathrm{a}^{+} \cdot 1=0 .
$$

Let $F(\lambda)$ be the induced $a$-module

$$
F(\lambda)=\operatorname{Ind}_{b}^{a} \mathbf{F}_{\lambda}=a \otimes_{b} \mathbf{F}_{\lambda} .
$$

By the defining relations of $\mathbf{a}$ we obtain an $\mathbf{F}$-linear isomorphism

$$
F(\lambda) \cong \mathfrak{a}^{-} .
$$


Since $\boldsymbol{a}^{-}$is abelian we may regard it as the algebra of polynomials in the variables $y_{1}, y_{2}, \cdots$. Then we see that $z$ acts on $a^{-} \cong \mathbb{F}\left[y_{1}, y_{2}, \cdots\right]$ by the multiplication of $\lambda, x_{p}$ acts by $\lambda \frac{\partial}{\partial y_{p}}$. By this realization we immediately have the following lemma.

Lemma $\mathfrak{B}_{0} \mathbb{1}$ 1. $F(\lambda)$ is an irreducible a-module.

Fix an skew-symmetric $(n+1) \times(n+1)$-matrix with integral coefficients $M=\left(m_{i j}\right)_{0 \leq i, j \leq n}$. We say that $\kappa \in \mathbb{Q}(q) *$ is generic with respect to $M$ if for any $k$ $\in \mathbb{Z}_{>0}$ the matrix $\left(\left[k\left\langle h_{\imath}, \alpha_{j}\right\rangle\right] \kappa^{\left.-k m_{t}\right)}\right)$ is invertible.

Note that if $n=1$ any $\kappa$ is not generic with respect to any $M$. Since the matrix $\left(\left[k\left\langle h_{\imath}, \alpha_{\jmath}\right\rangle\right]\right)_{0 \leq i, j \leq n}$ is invertible for $n>1$, there exists a generic $\kappa$ for $n>1$.

Lemma 3.1.2. (1) For a fixed $M$, we assume that $\kappa \in \mathbb{Q}(q) *$ is generic with respect to $M$ (in particular $n>1)$. Then $\mathscr{F}_{n}$ is an irreducible $S_{n}$-module.

(2) $\mathscr{F}_{1}$ is not irreducible.

Proof. (1) Set $G(k)=\left(g(k)_{\imath j}\right)\left(\left[k\left\langle h_{\imath}, \alpha_{j}\right\rangle\right] \kappa^{\left.-k m_{\imath}\right)}\right)$. Since $\kappa$ is generic with respect to $M$ there exists its inverse $G(k)^{-1}=\left(g(k)^{i j}\right)$ for any $k$. Note that by the definition $\sum_{0 \leq s \leq n} g(k){ }^{i s} g(k)_{s \jmath}=\delta_{\imath \jmath}$.

We set

$$
\widetilde{H}_{\imath k}= \begin{cases}\sum_{0 \leq s \leq n} \frac{k}{[k]} g(k){ }^{i s} H_{s, k}, & \text { for } k>0, \\ H_{\imath, k}, & \text { for } k<0\end{cases}
$$

Then we have

$$
\begin{gathered}
{\left[\widetilde{H}_{i, k}, \widetilde{H}_{j, l}\right]=\left[\widetilde{H}_{\imath,-k}, \widetilde{H}_{j,-l}\right]=0,} \\
{\left[\widetilde{H}_{\imath, k}, \widetilde{H}_{,-l}\right]=\delta_{k, l} \delta_{\imath \jmath},}
\end{gathered}
$$

for $k, l>0$. Since all $G(k)$ are regular, $\widetilde{H}_{\imath, k}(0 \leq i \leq n, k>0)$ generate $S_{n}^{+}$.

We shall use Lemma 3.1.1. Put $\mathbb{F}=\mathbb{Q}(q), \mathfrak{a}=S_{n}, \mathfrak{a}^{ \pm}=S_{n}^{ \pm}, \lambda=1, x_{p}=\widetilde{H}_{i, k}$ $(k>0), y_{r}=\tilde{H}_{, l}(l<0)$ where $p=(k-1)(n+1)+i+1$ and $r=(-l-1)(n+1)+j$ +1 .

Then it is clear that $F(1)=\mathscr{F}_{n}$. By Lemma 3.1.1 we conclude that $\mathscr{F}_{n}$ is an irreducible $S_{n}$-module.

(2) It is easy to see that $\kappa^{-k m_{01}} H_{0,-k}+H_{1,-k}$ is a central element of $S_{1}$ for each $k \in \mathbb{Z}_{>0}$. Therefore $\mathscr{F}_{1}$ has infinitely many singular vectors.

3.2. Construction of level $(\mathbb{1}, 0)$ modules. In this subsection we assume $\kappa=$ 1. Note that $1 \in \mathbb{Q}(q)$ is generic with respect to any $M$ with $n>1$. 
Note that one can rewrite $\bar{P}=\bigoplus_{i=2}^{n} \mathbf{Z} \overline{\alpha_{i}} \oplus \mathbf{Z} \bar{\Lambda}_{n}$. We introduce a twisted version of the group algebra $\mathbb{Q}(q)[\bar{P}]$ by $\mathbf{Z} / 2 \mathbf{Z}$. We denote it by $\mathbf{Q}(q)\{\bar{P}\}$. This is the $\mathbf{Q}(q)$-algebra generated by symbols $e^{\overline{\alpha_{2}}}, e^{\overline{\alpha_{3}}}, \ldots, e^{\overline{\alpha_{n}}}, e^{\overline{\Lambda_{n}}}$ which satisfy the following relations:

$$
\begin{aligned}
e^{\overline{\alpha_{t}}} e^{\overline{\alpha_{s}}} & =(-1)^{\left\langle\overline{h_{t}}, \overline{\left.\alpha_{s}\right\rangle}\right\rangle} e^{\overline{\alpha_{s}}} e^{\overline{\alpha_{t}}} \\
e^{\overline{\alpha_{t}}} e^{\overline{\Lambda_{n}}} & =(-1)^{\delta_{t} n} e^{\overline{\Lambda_{n}}} e^{\overline{\alpha_{t}}} .
\end{aligned}
$$

For $\bar{\alpha}=\sum_{1=2}^{n} m_{1} \overline{\alpha_{1}}+m_{n+1} \overline{\Lambda_{n}}$ we denote $e^{\bar{\alpha}}=\left(e^{\overline{\alpha_{2}}}\right)^{m_{2}}\left(e^{\overline{\alpha_{3}}}\right)^{m_{3}} \ldots\left(e^{\overline{\alpha_{n}}}\right)^{m_{n}}\left(e^{\overline{\Lambda_{n}}}\right)^{m_{n+1}}$. For example $e^{\overline{\alpha_{1}}}=e^{-2 \overline{\alpha_{2}}} e^{-3 \overline{a_{3}}} \cdots e^{-n \overline{\alpha_{n}}} e^{(n+1) \overline{\Lambda_{n}}}, e^{\overline{\Lambda_{t}}}=e^{-\overline{\alpha_{t+1}}} e^{-2 \overline{\alpha_{t+2}}} \cdots e^{-(n-i) \overline{\alpha_{n}}} e^{(n-1+1) \overline{\Lambda_{n}}}$ where $\bar{\Lambda}_{1}$ is the $i$-th fundamental weight. We denote $\bar{\alpha}_{0}=-\sum_{i=1}^{n} \bar{\alpha}_{\imath}$ and $\bar{h}_{0}=$ $-\sum_{t=1}^{n} \bar{h}_{\imath}$.

Note that $\left\langle h_{i}, \alpha_{\jmath}\right\rangle=\left\langle\bar{h}_{i}, \bar{\alpha}_{j}\right\rangle$ for $0 \leq i, j \leq n$.

We denote by $\mathbb{Q}(q)\{\bar{Q}\}$ the subalgebra of $\mathbb{Q}(q)\{\bar{P}\}$ generated by $e^{\overline{\alpha_{i}}}(1 \leq i$ $\leq n)$.

Set

$$
W(p)_{n}=\mathscr{F}_{n} \otimes \mathrm{Q}(q)\{\bar{Q}\}_{e^{\overline{\Lambda p}}}^{\text {for }} 1 \leq p \leq n
$$

and

$$
W(0)_{n}=\mathscr{F}_{n} \otimes \mathbf{Q}(q)\{\bar{Q}\} .
$$

We define the operators $H_{i, l}, e^{\bar{\alpha}}(\bar{\alpha} \in \bar{Q}), \partial \bar{\alpha}_{1}$ on $W(p)_{n}$ for $i=0,1, \ldots, n$ as follows: for $v \otimes e^{\bar{\beta}}=H_{21,-k 1} \ldots H_{1 N,-k_{N}} v_{0} \otimes e^{\bar{\beta}} \in W^{\prime}(p)_{n}$,

$$
\begin{aligned}
& H_{1, l}\left(v \otimes e^{\bar{\beta}}\right)=\left(H_{1, l} v\right) \otimes e^{\bar{\beta}}, \\
& e^{\bar{\alpha}}\left(v \otimes e^{\bar{\beta}}\right)=v \otimes e^{\bar{\alpha}} e^{\bar{\beta}} . \\
& \partial_{\overline{\alpha_{t}}}\left(v \otimes e^{\bar{\beta}}\right)=\left\langle\overline{h_{i}}, \bar{\beta}\right\rangle v \otimes e^{\bar{\beta}}, \\
& d\left(v \otimes e^{\bar{\beta}}\right)=\left(-\sum_{s=1}^{N} k_{s}-\frac{(\bar{\beta} \mid \bar{\beta})}{2}+\frac{\left(\overline{\Lambda_{p}} \mid \overline{\Lambda_{p}}\right)}{2}\right) v \otimes e^{\bar{\beta}} .
\end{aligned}
$$

We have the following lemma.

Lemma 3.2.1. As operators on $W(p)_{n}$,

$$
\begin{aligned}
& e^{\bar{\alpha}_{t} e^{\bar{\alpha}_{s}}}=(-1)^{\left\langle\bar{h}_{t}, \bar{\alpha}_{s}\right\rangle} e^{\overline{\alpha_{s}}} e^{\overline{\alpha_{t}}}, \\
& e^{\bar{\alpha}_{t}} q^{\partial \bar{\alpha}_{t}}=q^{\left\langle\bar{h}_{t}, \overline{\alpha_{\jmath}}\right\rangle} q^{\partial \bar{\alpha}_{t}} e^{\overline{\alpha_{t}}} .
\end{aligned}
$$

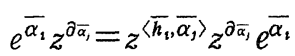

for $0 \leq i, j \leq n$. 
We introduce the following generating functions:

$$
\begin{aligned}
& E_{\imath}(z)=\sum_{k \in \mathbb{Z}} E_{\imath, k} z^{-k}, \\
& F_{\imath}(z)=\sum_{k \in \mathbf{Z}} F_{\imath, k} z^{-k} .
\end{aligned}
$$

Proposition 3.2.2. Let $c=1$ and $\kappa=1$. Then for each $p$ and $n$, the following action gives a $U_{q}^{\phi^{\prime}}\left(\mathrm{g}_{\text {tor }}\right)$-module structure on $W(p)_{n}$ :

$$
\begin{aligned}
& q^{\frac{1}{2} c \mapsto q^{\frac{1}{2}},} \\
& q^{d_{1}} \mapsto q^{d}, \\
& E_{\imath}(z) \mapsto \exp \left(\sum_{k \geq 1} \frac{H_{i,-k}}{[k]}\left(q^{-1 / 2} z\right)^{k}\right) \exp \left(\sum_{k \geq 1}-\frac{H_{2, k}}{[k]}\left(q^{1 / 2} z\right)^{-k}\right) e^{\bar{\alpha}_{t}} z^{\partial_{\overline{\alpha_{l}}}+1} \\
& F_{\imath}(z) \mapsto \exp \left(\sum_{k \geq 1}-\frac{H_{l,-k}}{[k]}\left(q^{1 / 2} z\right)^{k}\right) \exp \left(\sum_{k \geq 1} \frac{H_{l, k}}{[k]}\left(q^{-1 / 2} z\right)^{-k}\right) e^{-\bar{\alpha}_{z}} z^{-\bar{\alpha}_{t}+1} \\
& K_{1}^{+}(z) \mapsto \exp \left(\left(q-q^{-1}\right) \sum_{k \geq 1} H_{i, k} z^{-k}\right) q^{\hat{\sigma}_{\bar{\alpha}}}, \\
& K_{\imath}^{-}(z) \mapsto \exp \left(-\left(q-q^{-1}\right) \sum_{k \geq 1} H_{i,-k} z^{k}\right) q^{-\partial_{\bar{a}}}
\end{aligned}
$$

for $0 \leq i \leq n$.

The proof will be given in Appendix.

We have immediately the following lemma.

Lemma 3.2.3. The $U_{q}^{\phi^{\prime}}\left(\mathfrak{g}_{t o r}\right)$-module $W(p)_{n}$ is cyclic:

$$
W(p)_{n}=C_{q}^{\phi^{\prime}}\left(g_{t o r}\right)\left(v_{0} \otimes e^{\Lambda_{p}}\right) .
$$

Theorem 3.2.4. If $n>1$ then $W(p)_{n}$ is irreducible for any $p$.

Proof. Since $\mathscr{F}_{n}$ is irreducible with respect to the action of $S_{n}$. it is enough to show that for any non-zero $v=v_{0} \otimes \sum_{\bar{\alpha} \in \bar{Q}} a_{\bar{\alpha}} e^{\bar{\alpha}} e^{\bar{\Lambda}_{i}}\left(a_{\bar{\alpha}} \in \mathbb{Q}(q)\right)$ there exists $X \in$ $U_{q}^{\phi^{\prime}}\left(g_{t o r}\right)$ such that $X v=v_{0} \otimes e^{\overline{\Lambda_{p}}}$. Let $\overline{S_{n}}$ be the subalgebra of $S_{n}$ generated by $H_{n, l}$ $(1 \leq i \leq n, l \in \mathbb{Z} \backslash\{0\})$ and $q^{\frac{1}{2} c}$. Let $\overline{\mathscr{F}}_{n}$ be the $\overline{S_{n}}$-submodule of $\mathscr{F}_{n}$ generated by $v_{0}$ $\otimes e^{\bar{\Lambda}_{i}}$, and let $\overline{W(p)_{n}}=\overline{\mathscr{F}_{F}}{ }_{n} \otimes \mathbb{Q}(q)\{\bar{Q}\} e^{\overline{\Lambda_{p}}}$. As already known $\overline{W(p)_{n}}$ is an irreducible $U_{q}^{(1)}\left(\hat{\mathfrak{G l}}_{n+1}\right)$-module. It is obvious that $v \in \overline{W(p)_{n}}$. Therefore there exists $X \in U_{q}^{(1)}\left(\hat{\mathfrak{g}}_{n+1}\right) \subset U_{q}^{\phi^{\prime}}\left(\mathrm{g}_{t o r}\right)$ such that $X v=v_{0} \otimes e^{\overline{\Lambda_{p}}}$.

Remark 3.2.5. Since $\mathscr{F}_{1}$ is not irreducible as an $S_{1}$-module $W(p)_{1}$ is not 
irreducible.

Remark 3.2.6. Since $U_{q}^{(1)}\left(\widehat{\mathfrak{S l}}_{n+1}\right)$ and $U_{q}^{(2)^{\prime}}\left(\hat{\mathfrak{S}}_{n+1}\right)$ are subalgebras of $U_{q}^{\phi^{\prime}}\left(\mathfrak{g}_{t o r}\right)$ we can regard $W(p)_{n}$ as a $U_{q}^{(1)}\left(\widehat{\mathfrak{G l}}_{n+1}\right)$-module or as a $U_{q}^{(2))^{\prime}}\left(\hat{\mathfrak{G l}}_{n+1}\right)$ module. As a $U_{q}^{(1)}\left(\hat{\mathfrak{G}}_{n+1}\right)$-module, $W(p)_{n}$ is a level 1 module. On the other hand it is a level 0 module as a $U_{q}^{(2)^{\prime}}\left(\hat{\mathfrak{g l}}_{n+1}\right)$-module.

3.3. On the structure of level $(\mathbf{1 , 0})$ modules. In this subsection we will study the level $1 U_{q}\left(\hat{\mathfrak{s}}_{n+1}\right)$-module structure of $W(p)_{n}$.

Let $M$ be a $U_{q}^{(1)}\left(\hat{\mathfrak{S l}}_{n+1}\right)$-module. We can regard $M$ as a $U_{q}\left(\hat{\mathfrak{S I}}_{n+1}\right)$-module via a surjective homomorphism $U_{q}\left(\widehat{\mathfrak{S l}}_{n+1}\right) \rightarrow U_{q}^{(1)}\left(\hat{\mathfrak{G l}}_{n+1}\right)$. As a $U_{q}\left(\hat{\mathfrak{G l}}_{n+1}\right)$-module, we denote the character of $M$ by $\mathrm{ch}_{M}$.

Let $L\left(\Lambda_{p}\right)$ be the irreducible highest weight $U_{q}\left(\hat{\mathfrak{G}}_{n+1}\right)$-module with highest weight $\Lambda_{p}$. Note that the following identity holds:

$$
\operatorname{ch}_{L\left(\Lambda_{p}\right)}=\frac{e^{\Lambda_{p}} \sum_{\alpha \in \bar{Q}} e^{\alpha-\left(\frac{1}{2}(\alpha \mid \alpha)-\left(\alpha \mid \overline{\Lambda_{p}}\right)\right) \delta}}{\varphi\left(e^{-\delta}\right)^{n}} .
$$

Here $\varphi(x)=\prod_{k>0}\left(1-x^{k}\right)$.

We denote $\delta$ by the null root of $U_{q}\left(\hat{\mathfrak{G}}_{n+1}\right)$.

By the definition of $W(p)_{n}$ and (3.2.1) it is immediate to see the following proposition.

Proposition 3.3.1. As a $L_{q}^{\top}\left(\hat{\mathfrak{S l}}_{n+1}\right)$-module, we have

$$
\begin{aligned}
c h_{W(p)_{n}} & =\frac{e^{\Lambda_{p}} \sum_{\alpha \in \bar{Q} e^{\alpha-\left(\frac{1}{2}(\alpha \mid \alpha)-\left(\alpha \mid \overline{\Lambda_{p}}\right)\right) \delta}}^{\varphi\left(e^{-\delta}\right)^{n+1}}}{}=\frac{c h_{L\left(\Lambda_{p}\right)}}{\varphi\left(e^{-\delta}\right)} .
\end{aligned}
$$

Lemma 3.3.2. For each $l \in \mathbb{Z} \backslash\{0\}$ there exist $\widehat{H}_{l}=\sum_{l=0 a_{\imath, l}}^{n} H_{\imath, l}\left(a_{\imath, l} \in \mathbb{Q}(q)\right)$ such that

$$
\left[\widehat{H}_{l,} H_{\jmath, k}\right]=0
$$

for any $1 \leq j \leq n$ and $k \in \mathbb{Z} \backslash\{0\}$. Moreover such $\widehat{H}_{l}$ is unique up to scalar.

Proof. Note that $\kappa=1$. The rank of $n \times(n+1)$-matrix $\left(\left[l\left\langle h_{i}, \alpha_{\jmath}\right\rangle\right]\right)_{1 \leq \imath \leq n, 0 \leq \jmath \leq n}$ is equal to $n$. The lemma follows form this fact immediately.

By the definition of $\widehat{H}_{l}$ we have

$$
\left[\widehat{H}_{k}, \widehat{H}_{l}\right]=\delta_{k+l, 0} \gamma_{k} .
$$


where $\gamma_{k} \in \mathbb{Q}(q)$. We fix a normalization of $\hat{H}_{l}$ by putting $\gamma_{k}=1$ for all $k$. $W(p)_{n}$.

Let $\widehat{S}_{n}$ be the subalgebra of $S_{n}$ generated by $\widehat{H}_{l}$. By the definition, $\widehat{S}_{n}$ acts on

The following two lemmas are easy to see.

Lemma 3.3.3. For $l>0, \widehat{H}_{l}\left(v_{0} \otimes e^{\bar{\beta}}\right)=0$.

Lemma 3.3.4. The action of $\left(I_{q}^{\prime}\left(\hat{\mathfrak{S}}_{n+1}\right)\right.$ on $W(p)_{n}$ commutes with the action of $\widehat{S_{n}}$.

Let $\widehat{S}_{n}^{-}$be the subalgebra of $\widehat{S}_{n}$ generated by $\widehat{H}_{l}(l<0)$.

Proposition 3.3.5. As $U_{q}\left(\widehat{\mathfrak{G l}}_{n+1}\right)$-module

$$
W(p)_{n} \cong L\left(\Lambda_{p}\right)^{\oplus \infty} \text {. }
$$

Proof. Set deg $\left(\widehat{H}_{k}\right)=k$. Let $M_{k}=M_{k}\left(\widehat{H}_{-1}, \widehat{H}_{-2}, \ldots\right)$ be a monomial of degree $k$ in variables $\widehat{H}_{-1}, \widehat{H}_{-2}, \ldots$. Then by the above two lemmas, $M_{k} v_{0} \otimes e^{\bar{\Lambda}_{p}}$ is a singular vector of $U_{q}^{(1)}\left(\hat{\mathfrak{S l}}_{n+1}\right)$-module $W(p)_{n}$. Let $W_{M_{k}}$ be the $U_{q}\left(\hat{\mathfrak{S}}_{n+1}\right)$-submodule which is generated by $M_{k} v_{0} \otimes e^{\overline{\Lambda_{p}}}$. Then by the definition of the action of $U_{q}\left(\widehat{\mathfrak{S l}}_{n+1}\right)$ on $W(p)_{n}$, we have

$$
W_{M_{k}} \cong L\left(\Lambda_{p}-k \delta\right) \cong L\left(\Lambda_{p}\right) .
$$

The vectors $\left\{M_{k} v_{0} \otimes e^{\overline{T_{p}}}\right\}$ are linearly independent. The number of the monomials of degree $k$ is equal to the $k$-th partition number $p(k)$. Therefore there is a $U_{q}^{(1)}\left(\widehat{\mathscr{Q}}_{n+1}\right)$-submodule $W$ of $W(p)_{n}$ which is isomorphic to $\bigoplus_{k \geq 0} L\left(\Lambda_{p}\right.$ $-k \delta)^{\oplus p(k)}$. By Proposition 3.3.1 it coincides with $W(p)_{n}$. This completes proof.

By Lemma 3.3.4 and the proof of Proposition 3.3.5, the following corollary follows immediately.

Corollary 3.3.6. As $U_{q}^{\prime}\left(\widehat{\mathfrak{G l}}_{n+1}\right) \otimes \widehat{S}_{n}$-module $W(p)_{n}$ is isomorphic to $L\left(\Lambda_{p}\right)$ $\otimes \widehat{S}_{n}^{-}$.

3.4. Construction of level $(1, \mathbb{1})$ modules. We introduce a twisted version of the group algebra $\mathbb{Q}(q)[Q]$ by $\mathbb{Z} \backslash 2 \mathbb{Z}$. We denoted it by $\mathbb{Q}(q)\{Q\}$. This is the $\mathbb{Q}(q)$-algebra generated by symbols $e^{\alpha_{0}}, e^{\alpha_{1}}, \ldots, e^{\alpha_{n}}$ which satisfy the following relations:

$$
e^{\alpha_{t}} e^{\alpha_{s}}=(-1)^{\left\langle h_{t}, \alpha_{s}\right\rangle} e^{\alpha_{t}} e^{\alpha_{t}} .
$$

Similarly to $\S 3.2$, we denote $e^{\alpha}=\left(e^{\alpha_{0}}\right)^{m_{0}}\left(e^{\alpha_{1}}\right)^{m_{1}} \ldots\left(e^{\alpha_{n}}\right)^{m_{n}}$ for $\alpha=\sum_{i=0}^{n} m_{i} \alpha_{i} \in Q$. 
Let

$$
V(p)_{n}=\mathscr{F}_{n} \otimes \mathbf{Q}(q)\{Q\} e^{\Lambda_{p}}
$$

Here we regard $e^{\Lambda_{p}}$ only a symbol indexed by $p$.

We define the operators $H_{i, l}(0 \leq i \leq n, l \neq 0) . e^{a}(\alpha \in Q), \partial_{\alpha_{i}}$ and $z^{H_{i, 0}}(0 \leq i$ $\left.\leq_{n}\right)$ on $V(p)_{n}$ as follows:

for $v \otimes e^{\beta} e^{\Lambda_{p}}=H_{i_{1},-k_{1}} \ldots H_{i_{N},-k N} v_{0} \otimes e^{\beta} e^{\Lambda_{p}} \in V(p)_{n}\left(\beta=\sum_{k=0}^{n} m_{k} \alpha_{k} \in Q\right)$,

$$
\begin{aligned}
& H_{i, l}\left(v \otimes e^{\beta} e^{\Lambda_{p}}\right)=\left(H_{i, l}\right) \otimes e^{\beta} e^{\Lambda_{p}}, \\
& e^{\alpha}\left(v \otimes e^{\beta} e^{\Lambda_{p}}\right)=v \otimes\left(e^{\alpha} e^{\beta}\right) e^{\Lambda_{p}}, \\
& \partial_{\alpha_{l}}\left(v \otimes e^{\beta} e^{\Lambda_{p}}\right)=\left\langle h_{\imath}, \beta+\Lambda_{p}\right\rangle v \otimes e^{\beta} e^{\Lambda_{p}}, \\
& z^{H_{t, 0}}\left(v \otimes e^{\beta} e^{\Lambda_{p}}\right)=z^{\left\langle h_{t}, \beta+\Lambda_{p}\right\rangle} \kappa^{\frac{1}{2} \sum_{k=0}^{n} / h_{t}, m_{L} \alpha_{k \prime} m_{t h}} v \otimes e^{\beta} e^{\Lambda_{p}} . \\
& d_{1}\left(v \otimes e^{\beta} e^{\Lambda_{p}}\right)=\left(-\sum_{s=1}^{N} k_{s}-\frac{(\beta \mid \beta)}{2}-\left(\beta \mid \Lambda_{p}\right)\right) \imath \otimes e^{\beta} e^{\Lambda_{p}}, \\
& d_{2}\left(v \otimes e^{\beta} e^{\Lambda_{p}}\right)=m_{0}\left(v \otimes e^{\beta} e^{\Lambda_{p}}\right) .
\end{aligned}
$$

The following lemma is easy.

Lemma 3.4.1. As operators on $V(p)_{n}$,

$$
\begin{aligned}
& e^{\alpha_{t}} e^{\alpha_{s}}=(-1)^{\left\langle h_{t}, \alpha,\right\rangle} e^{\alpha_{s}} e^{\alpha_{t}} \\
& q^{\partial_{\alpha_{1}}} e^{\alpha_{J}}=q^{\left\langle h_{1}, \alpha_{j}\right\rangle} e^{\alpha} q^{\partial_{\alpha_{1}}} \\
& z^{H_{t, 0}} e^{\alpha_{f}}=z^{\left\langle h_{h} \cdot \alpha_{,}\right\rangle} \kappa^{\frac{1}{2}\left\langle h_{t}, \alpha,\right\rangle m_{t}} e^{\alpha_{1}} z^{H_{t, 0}} .
\end{aligned}
$$

Proposition 3.4.2. Assume $c=1$ then for each $p$ and $n$, the following action gives a $U_{q}\left(g_{t o r}\right)$-module structure on $V(p)_{n}$ :

$$
\begin{aligned}
& q^{\frac{1}{2} c} \mapsto q^{\frac{1}{2}}, \\
& q^{d_{1}} \mapsto q^{d_{1}}, \\
& q^{d_{2}} \mapsto q^{d_{2}}, \\
& E_{t}(z) \mapsto \exp \left(\sum_{k \geq 1} \frac{H_{l,-k}}{[k]}\left(q^{-1 / 2} z\right)^{k}\right) \exp \left(\sum_{k \geq 1}-\frac{H_{t, k}}{[k]}\left(q^{1 / 2} z\right)^{-k}\right) e^{\alpha_{i}} z^{H_{t, 0+1}}, \\
& F_{2}(z) \mapsto \exp \left(\sum_{k \geq 1}-\frac{H_{i,-k}}{[k]}\left(q^{1 / 2} z\right)^{k}\right) \exp \left(\sum_{k \geq 1} \frac{H_{l, k}}{[k]}\left(q^{-1 / 2} z\right)^{-k}\right) e^{-\alpha_{i}} z^{-H_{t, 0+1}}, \\
& K_{i}^{+}(z) \mapsto \exp \left(\left(q-q^{-1}\right) \sum_{k \geq 1} H_{t, k} z^{-k}\right) q^{\partial_{\alpha_{i}}} .
\end{aligned}
$$




$$
K_{i}^{-}(z) \mapsto \exp \left(-\left(q-q^{-1}\right) \sum_{k \geq 1} H_{\imath,-k} z^{k}\right) q^{-\partial_{\alpha \prime}}
$$

for $0 \leq_{i} \leq_{n}$

The proof will be given in Appendix.

It is easy to see the following lemma.

Lemma 3.4.\&. $V(p)_{n}$ is a cyclic $U_{q}\left(\mathfrak{g}_{t o r}\right)$-modulc: $V(p)_{n}=U_{q}\left(\mathfrak{g}_{t o r}\right)\left(v_{0} \otimes e^{\Lambda_{p}}\right)$.

Lemma 3.4.4. $V(p)_{n}$ has level 1 as a $U_{q}^{(1)}\left(\hat{\mathfrak{S}}_{n+1}\right)$-module and as a $U_{q}^{(2)}\left(\hat{\mathfrak{s l}}_{n+1}\right)$-module.

Proof. It is clear that $V(p)_{n}$ is a level $1 U_{q}^{(1)}\left(\hat{\mathfrak{G}}_{n+1}\right)$-module. The center of $U_{q}^{(2)}\left(\hat{\mathfrak{g l}}_{n+1}\right)$ is $\prod_{k=0}^{n} K_{\imath}$. By the definition it acts as the scalar $q$ on $V(p)_{n}$.

\section{\$4. $O \mathrm{On} \mathbb{U}_{q}\left(\mathfrak{S L}_{2, t o r}\right)$}

4.1. In this section we assume that $\mathfrak{g}=\mathfrak{g l}_{2}$. We shall try to find finitely many generators of $U_{q}\left(\mathfrak{B l}_{2, t o r}\right)$.

Let

$$
\begin{gathered}
E_{i}=E_{i, 0}, F_{\imath}=F_{i, 0}, q^{ \pm h_{t}}=K_{\imath}^{ \pm}, \text {for } i=0,1, \\
E_{-1}=F_{0,1}, K_{0}^{-}, F_{-1}=K_{0}^{ \pm} E_{0,-1}, q^{ \pm h-1}=q^{ \pm c} K_{0}^{\mp} .
\end{gathered}
$$

Proposition 4.1.1. $U_{q}\left(\mathfrak{S l}_{2, t o r}\right)$ is generated by $E_{l}, F_{\imath}, q^{ \pm h_{\imath}}(i=-1,0,1), q^{ \pm \frac{1}{2} c}$. $q^{ \pm d_{1}} \cdot q^{ \pm d_{2}}$.

Proof. Let $\mathscr{A}$ be the subalgebra of $L_{q}\left(\mathfrak{S l}_{2, \text { tor }}\right)$ generated by $E_{\imath}, F_{i}, q^{ \pm h_{1}}(i=$ $-1,0,1) \cdot q^{ \pm \frac{1}{2} c} \cdot q^{ \pm d_{1}}, q^{ \pm d 2}$. By the definition we have $E_{0,-1}=q^{-h_{0}} F_{-1}$ and $F_{0,1}=$ $E_{-1} q^{h_{0}}$. Since

$$
\begin{aligned}
{\left[E_{0,0}, F_{0,1}\right] } & =\frac{1}{q-q^{-1}} q^{-\frac{1}{2} c} K_{0,1}^{+} \\
& =q^{-\frac{1}{2} c} q^{h_{0}} H_{0,1}
\end{aligned}
$$

and

$$
\begin{aligned}
{\left[E_{0,-1}, F_{0,0}\right] } & =-\frac{1}{q-q^{-1}} q^{\frac{1}{2} c} K_{0,-1}^{-} \\
& =q^{\frac{1}{2} c} q^{-h_{0}} H_{0,-1} .
\end{aligned}
$$

We deduce $H_{0,1}$, and $H_{0,-1} \in \mathscr{A l}$. We recall $(2.2 .7)$ 


$$
\begin{aligned}
& {\left[H_{\imath, k} E_{j, l}\right]=\frac{1}{k}\left[k\left\langle h_{\imath}, \alpha_{j}\right\rangle\right] q^{-\frac{1}{2}|k| c} \kappa^{-k m_{\imath} v} E_{j, k+l},} \\
& {\left[H_{l, k}, F_{\jmath, l}\right]=-\frac{1}{k}\left[k\left\langle h_{i}, \alpha_{\jmath}\right\rangle\right] q^{\frac{1}{2}|k| c} \kappa^{-k m_{u}} F_{\jmath, k+l} .}
\end{aligned}
$$

By these formulas we have $E_{\imath, k}, F_{\imath, k} \in \mathscr{A}$ for $i=0,1, k \in \mathbb{Z}$ in inductively.

On the other hand we know

$$
\begin{aligned}
{\left[E_{0,1}, F_{0,1}\right] } & =\frac{1}{q-q^{-1}} q^{-\frac{1}{2} c} K_{0,2}^{+} \\
& =\frac{1}{2}\left(q-q^{-1}\right) H_{0,1}^{2}+H_{0,2}
\end{aligned}
$$

Therefore we get $H_{0,2} \in \mathscr{A}$. Similary we have $H_{1, l} \in \mathscr{A}$ for any $i, l$.

This completes the proof.

Lemma 4.1.2. The following relations hold in $U_{q}\left(\mathfrak{g l}_{2, t o r}\right)$ :

$$
\begin{gathered}
{\left[q^{ \pm h_{t}}, q^{ \pm h_{\jmath}}\right]=0,} \\
{\left[q^{ \pm d_{t}}, q^{ \pm h_{\jmath}}\right]=0,} \\
q^{d_{1}} E_{j} q^{-d_{1}}=q^{\delta_{\jmath-1}} E_{\jmath}, \\
q^{d_{1}} F_{\jmath} q^{-d_{1}}=q^{-\delta_{\jmath,-1}} F_{\jmath}, \\
q^{d_{2}} E_{\jmath} q^{-d_{2}}=q^{-1+\delta_{s, 1}} E_{\jmath}, \\
q^{d_{2}} F_{\jmath} q^{-d_{2}}=q^{1-\delta_{\jmath, 1}} F_{j}, \\
q^{h_{t}} E_{\jmath} q^{-h_{t}}=q^{a_{t}} E_{\jmath}, \\
q^{h_{t}} F_{\jmath} q^{-h_{t}}=q^{-a_{t} F_{\jmath},},
\end{gathered}
$$

where

$$
\left(a_{i j}\right)_{-1 \leq i, j \leq 1}=\left(\begin{array}{ccc}
2 & -2 & 2 \\
-2 & 2 & -2 \\
2 & -2 & 2
\end{array}\right) \text {. }
$$

$$
\left[E_{\imath}, F_{\jmath}\right]=\delta_{\imath} \frac{q^{h_{\imath}}-q^{-h_{\imath}}}{q-q^{-1}} . \text { for }|i-j| \leq 1
$$

(4.1.7) $\quad E_{-1}^{3} F_{1}-q^{-2}[3] E_{-1}^{2} F_{1} E_{-1}+q^{-4}[3] E_{-1} F_{1} E_{-1}^{2}-q^{-6} F_{1} E_{-1}^{3}=0$,

(4.1.8) $\quad E_{1}^{3} F_{-1}-q^{2}[3] E_{1}^{2} F_{-1} E_{1}+q^{4}[3] E_{1} F_{-1} E_{1}^{2}-q^{6} F_{-1} E_{1}^{3}=0$,

(4.1.9) $\quad F_{-1}^{3} E_{1}-q^{-2}[3] F_{-1}^{2} E_{1} F_{-1}+q^{-4}[3] F_{-1} E_{1} F_{-1}^{2}-q^{-6} E_{1} F_{-1}^{3}=0$, 


$$
F_{1}^{3} E_{-1}-q^{2}[3] F_{1}^{2} E_{-1} F_{1}+q^{4}[3] F_{1} E_{-1} F_{1}^{2}-q^{6} E_{-1} F_{1}^{3}=0,
$$

$$
E_{\imath}^{3} E_{\jmath}-[3] E_{\imath}^{2} E_{j} E_{\imath}+[3] E_{\imath} E_{j} E_{i}^{2}-E_{\jmath} E_{i}^{3}=0, \quad \text { for }|i-j|=1 \text {, }
$$

(4.1.12) $F_{i}^{3} F_{j}-[3] F_{i}^{2} F_{j} F_{i}+[3] F_{\imath} F_{j} F_{i}^{2}-F_{j} F_{i}^{3}=0, \quad$ for $|i-j|=1$,

$$
E_{-1} E_{1}-q^{2} E_{1} E_{-1}=0 \text {, }
$$

$$
F_{-1} F_{1}-q^{2} F_{1} F_{-1}=0 \text {. }
$$

Proof. By the definition of $U_{q}\left(\mathfrak{g}_{t o r}\right)$ and $E_{i}, F_{i}$ and $q^{h_{i}}$ it is easy to check these relations.

Let $\mathcal{U}$ be an associative algebra over $\mathbb{Q}(q)$ generated by $E_{i}, F_{i}, q^{ \pm h_{t}}(i=$ $-1,0,1), q^{ \pm \frac{1}{2} c}, q^{ \pm d_{1}}, q^{ \pm d_{2}}$ with relations $(4.1 .1)-(4.1 .14)$. Then we have.

Corollary 4.1.3. There is a canonical surjective algebra homomorphism $\Psi: U \rightarrow$ $U_{q}\left(\mathfrak{S l}_{2, t o r}\right)$.

Remark 4.1.4. $\Psi$ has a highly nontrivial kernel. It is important to determine it. For example the following formulas holds in $U_{q}\left(\mathfrak{G l}_{2, t o r}\right)$ :

$$
\begin{gathered}
\kappa^{m 01} E_{0,0} E_{1,-1}-q^{-2} \kappa^{m 01} E_{1,-1} E_{0,0}=q^{-2} E_{0,-1} E_{1,0}-E_{1,0} E_{0,-1}, \\
E_{0,-1}=q^{-h_{0}} F_{-1}
\end{gathered}
$$

and

$$
E_{1,-1}=\frac{\kappa^{-m_{01}}}{[-2]}\left[F_{-1} F_{0}-q^{-2} F_{0} F_{-1}, E_{1}\right]
$$

Therefore we have

$$
\begin{aligned}
X= & \frac{1}{[-2]} E_{0}\left[F_{-1} F_{0}-q^{-2} F_{0} F_{-1}, E_{1}\right]-\frac{q^{-2}}{[-2]}\left[F_{-1} F_{0}-q^{-2} F_{0} F_{-1}, E_{1}\right] E_{0} \\
& -q^{-2} q^{-h_{0}} F_{-1} E_{1}-E_{1} q^{-h_{0}} F_{-1} \\
= & 0
\end{aligned}
$$

in $U_{q}\left(\mathfrak{B l}_{2, t o r}\right)$. Thus $X \in \operatorname{Ker} \Psi$. But, as an element of $\mathcal{U}, X$ is not equal to 0 .

4.2. Let

$$
E_{0 *}=F_{1,1} K_{1}^{-}, \quad F_{0 *}=K_{1}^{+} E_{1,-1}, \quad q^{ \pm h_{0 *}}=q^{ \pm c} K_{1}^{\mp} .
$$

Proposition 4.2.1. The subalgebra generated by $E_{\imath}, F_{\imath}$, and $q^{h_{i}}$ for $i=0,1$, $E_{0^{*}}, F_{0^{*}}, q^{h_{0^{4}}}, q^{ \pm c}, q^{ \pm d_{1}}, q^{ \pm d_{2}}$, is equal to $U_{q}\left(\mathfrak{B l}_{2, t o r}\right)$. That is, they are generators of 
$U_{q}\left(\mathfrak{B l}_{2, t o r}\right)$. Moreover these generators satisfy relations similar to the ones in Lemma 4.1.2.

Proof. This proposition is proved in the same way as Proposition 4.1.1 and Lemma 4.1.2.

We have immediately the following lemma.

Lemma 4.2.2. Let $U_{q}^{(1)}$ be the subalgebras generated by $E_{i}, F_{i}, q^{h_{t}}$ for $i=1,0^{*}$ and $q^{ \pm\left(d_{1}+d_{2}\right)}, U_{q}^{(2)}$ the subalgebras generated by $E_{\imath}, F_{1}, q^{h_{1}}$ for $i=0,1$ and $q^{ \pm\left(d_{1}+d_{2}\right)}$, $U_{q}^{(3)}$ the subalgebras generated by $E_{\imath}, F_{\imath}, q^{ \pm h_{t}}$ for $i=0^{*},-1$ and $q^{ \pm\left(d_{1}+d_{2}\right)}$, and $U_{q}^{(4)}$ the subalgebras generated by $E_{1}, F_{1}, q^{ \pm h_{t}}$ for $i=0,-1$ and $q^{ \pm\left(d_{1}+d_{2}\right)}$. Then $L_{q}^{\left(t^{(1)}\right.}(i=$ $1,2,3,4)$ are isomorphic to $U_{q}\left(\widehat{\mathfrak{G}}_{2}\right)$.

Those four algebras are schematically visualized by Fig. 1.

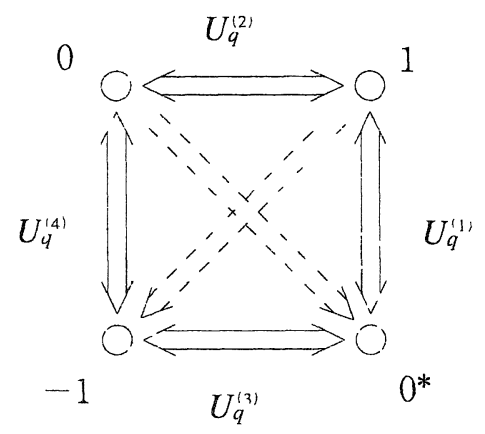

Fig. 1.

Let $U_{q}\left(\mathfrak{S I}_{2}\right)_{(\imath)}\left(i=-1,0,1,0^{*}\right)$ be the subalgebra of $U_{q}\left(\mathfrak{g l}_{2, t o r}\right)$ generated by $E_{\imath}, F_{\imath}$, $q^{ \pm h_{i}}$. All $U_{q}\left(\mathfrak{S l}_{2,(t)}\right)\left(i \in\left\{-1,0,1,0^{*}\right\}\right)$ are isomorphic to $U_{q}\left(\mathfrak{G l}_{2}\right)$. The upper left circle in Fig. 1 means $U_{q}\left(\mathfrak{g l}_{2}\right)_{(0)}$, the upper right one means $U_{q}\left(\mathfrak{g l}_{2}\right)_{(1)}$, the lower left one means $U_{q}\left(\mathfrak{S I}_{2}\right)_{(-1)}$ and the lower right one means $U_{q}\left(\mathfrak{g l}_{2}\right)_{(0 *)}$. The diagram

$$
\mathrm{O}^{2} \Longleftrightarrow \mathrm{O}^{\prime} \quad\left(i, j \in\left\{-1,0,1,0^{*}\right\}\right)
$$

means the algebra generated by $U_{q}\left(\mathfrak{G I}_{2}\right)_{(\imath)}$ and $U_{q}\left(\mathfrak{S I}_{2}\right)_{(\jmath)}$ is isomorphic to $U_{q}\left(\widehat{\mathfrak{g l}}_{2}\right)$. For example $\mathrm{O}^{0} \Longleftrightarrow \mathrm{O}^{1}$ means the algebra generated by $U_{q}\left(\mathfrak{G l}_{2}\right)_{(0)}$ and $U_{q}\left(\mathfrak{S l}_{2}\right)_{(-1)}$ which we call $U_{q}^{(2)}$ is isomorphic to $U_{q}\left(\hat{\mathfrak{G}}_{2}\right)$. The meaning of the diagram

$$
O^{2}=====\Rightarrow O^{\prime}
$$

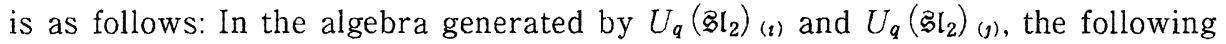
relations hold

$$
q^{h_{1}} E_{\jmath} q^{-h_{\imath}}=q^{2} E_{j}, \quad q^{h_{\jmath}} E_{i} q^{-h_{s}}=q^{2} E_{\imath},
$$




$$
\begin{gathered}
q^{h_{\imath}} F_{j} q^{-h_{i}}=q^{-2} F_{j}, \quad q^{h_{j}} F_{i} q^{-h_{s}}=q^{-2} F_{\imath} . \\
E_{i}^{3} F_{\jmath}-q^{-2}[3] E_{i}^{2} F_{j} E_{i}+q^{-4}[3] E_{\imath} F_{j} E_{i}^{2}-q^{-6} F_{j} E_{i}^{3}=0, \\
E_{j}^{3} F_{i}-q^{2}[3] E_{j}^{2} F_{\imath} E_{j}+q^{4}[3] E_{\jmath} F_{\imath} E_{j}^{2}-q^{6} F_{\imath} E_{j}^{3}=0, \\
F_{\imath}^{3} E_{j}-q^{-2}[3] F_{\imath}^{2} E_{j} F_{i}+q^{-4}[3] F_{i} E_{\jmath} F_{\imath}^{2}-q^{-6} E_{j} F_{i}^{3}=0, \\
F_{j}^{3} E_{i}-q^{2}[3] F_{j}^{2} E_{\imath} F_{\jmath}+q^{4}[3] F_{\jmath} E_{\imath} F_{j}^{2}-q^{6} E_{\imath} F_{j}^{3}=0, \\
E_{1} E_{\jmath}-q^{2} E_{j} E_{\imath}=0, \\
F_{\imath} F_{j}-q^{2} F_{j} F_{\imath}=0 .
\end{gathered}
$$

\section{Appendix $\mathbb{A}$.}

A.1. Proof of Proposition 3.2.2 and 3.4.2. For the proof we rewrite the defining relation $U_{q}\left(g_{t o r}\right)$ generating function level. $q^{ \pm \frac{1}{2} c} \quad$ are central.

$$
K_{\imath}^{+} K_{1}^{-}=K_{\imath}^{-} K_{\imath}^{+}=1,
$$

$$
K_{i}^{ \pm}(z) K_{\jmath}^{ \pm}(w)=K_{\jmath}^{ \pm}(w) K_{i}^{ \pm}(z)
$$

(A.1.4)

$$
\theta_{-\left\langle h_{t}, \alpha,\right\rangle}\left(q^{-c} \kappa^{-m_{t} z} \frac{z}{w}\right) K_{\imath}^{-}(z) K_{j}^{+}(w)=\theta_{-\left\langle h_{t}, \alpha,\right\rangle}\left(q^{c} \kappa^{-m_{u}} \frac{z}{w}\right) K_{j}^{+}(w) K_{\imath}^{-}(z)
$$

$$
q^{d_{1}} K_{j}^{ \pm}(z) q^{-d_{1}}=K_{j}^{ \pm}\left(q^{-1} z\right),
$$

$$
\left[q^{d_{2}}, K_{j}^{ \pm}(z)\right]=0
$$

$$
\begin{aligned}
& q^{d_{1}} E_{j}(z) q^{-d_{1}}=E_{j}\left(q^{-1} z\right) . \\
& q^{d_{1}} F_{j}(z) q^{-d_{1}}=F_{j}\left(q^{-1} z\right) .
\end{aligned}
$$

$$
\begin{gathered}
q^{d_{2}} E_{\jmath}(z) q^{-d_{2}}=q^{\delta_{\jmath} 0} E_{\jmath}(z), \\
q^{d_{2}} F_{,}(z) q^{-d_{2}}=q^{-\delta \delta_{0}} F_{\jmath}(z),
\end{gathered}
$$

$$
\begin{gathered}
K_{i}^{+}(z) E_{\jmath}(w)=\theta_{-\left\langle h_{t}, \alpha,\right\rangle}\left(q^{-\frac{1}{2} c} \kappa^{-m_{t}} \frac{w}{z}\right) E_{\jmath}(w) K_{i}^{+}(z) \\
K_{t}^{-}(z) E_{j}(w)=\theta_{\left\langle h_{t}, \alpha,\right\rangle}\left(q^{-\frac{1}{2} c} \kappa^{m_{t \jmath}} \frac{z}{w}\right) E_{j}(w) K_{\imath}^{-}(z) \\
K_{i}^{+}(z) F_{j}(w)=\theta_{\left\langle h_{t}, \alpha,\right\rangle}\left(q^{\frac{1}{2} c} \kappa^{-m_{t}, \frac{w}{z}}\right) F_{j}(w) K_{\imath}^{+}(z)
\end{gathered}
$$




$$
K_{i}^{-}(z) F_{j}(w)=\theta_{-\left\langle h_{t}, \alpha\right\rangle}\left(q^{\frac{1}{2} c} \kappa^{m_{\imath},} \frac{z}{w}\right) F_{j}(w) K_{\imath}^{-}(z)
$$

(A.1.10)

$$
\left[E_{i}(z), F,(w)\right]=\delta_{i, j} \frac{1}{q-q^{-1}}\left\{\delta\left(q^{c} \frac{w}{z}\right) K_{i}^{+}\left(q^{\frac{1}{2} c} w\right)-\delta\left(q^{c} \frac{z}{w}\right) K_{i}^{-}\left(q^{\frac{1}{2} c} z\right)\right\}
$$

$$
\begin{gathered}
\left(\kappa^{m_{\imath} z} z-q^{\left\langle h_{\imath}, \alpha\right\rangle} w\right) E_{\imath}(z) E_{j}(w)=\left\langle q^{\left\langle h_{\imath}, \alpha_{\jmath}\right\rangle} \kappa^{m_{t}} z-w\right) E_{\jmath}(w) E_{\imath}(z) \\
\left(\kappa^{m_{\imath} z} z-q^{-\left\langle h_{t}, \alpha,\right\rangle} w\right) F_{\imath}(z) F_{j}(w)=\left(q^{-\left\langle h_{\imath}, \alpha_{\imath}\right\rangle} \kappa^{m_{\imath \jmath}} z-w\right) F_{\jmath}(w) F_{\imath}(z)
\end{gathered}
$$

$(\mathrm{A} .1 .12)$

$$
\begin{gathered}
\sum_{\sigma \in \Xi_{m}} \sum_{r=0}^{m}(-1)^{r}\left[\begin{array}{c}
m \\
r
\end{array}\right]_{q} E_{\imath}\left(z_{\sigma(1)}\right) \cdots E_{\imath}\left(z_{\sigma(r)}\right) E_{j}(w) E_{\imath}\left(z_{\sigma(r+1)}\right) \cdots E_{\imath}\left(z_{\sigma(m)}\right)=0 \\
\sum_{\sigma \in \subseteq_{m}} \sum_{r=0}^{m}(-1)^{r}\left[\begin{array}{c}
m \\
r
\end{array}\right]_{q} F_{i}\left(z_{\sigma(1)}\right) \cdots F_{\imath}\left(z_{\sigma(r)}\right) F_{j}(u) F_{i}\left(z_{\sigma(r+1)}\right) \cdots F_{\imath}\left(z_{\sigma(m)}\right)
\end{gathered}
$$

where $i \neq j$ and $m=1-\left\langle h_{\imath}, \alpha_{j}\right\rangle$.

In these formulas we denote $\theta_{m}(z)=\frac{z q^{m}-1}{z-q^{m}}$ for $m \in \mathbb{Z} . \delta(z)=\sum_{k \in \mathbf{Z}} z^{k}$.

If Proposition 3.4.2 holds, then, from Lemma 3.2.1 and 3.4.1, we have

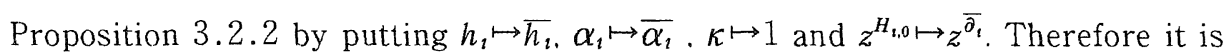
enough to show Proposition 3.4.2.

The relations (A.1.1), (A.1.2) and (A.1.3) are trivial. (A.1.4) is just the commutation relations of Heisenberg algebra $S_{n}$. Therefore, by the definition of $V(p)_{n}$, it is clear that (A.1.4) holds. The relations (A.1.5), (A.1.6) immediately follows from the definition of $d_{1}$ and $d_{2}$.

Let us show (A.1.7) and (A.1.8). Take $v \otimes e^{\beta} c^{\Lambda_{p}} \in V(p)_{n}$ where $\beta=$ $\sum_{k=0}^{n} m_{k} \alpha_{k} \in Q$. Then we have

$$
\begin{aligned}
& q^{d_{1}} e^{ \pm \alpha_{j}} z^{ \pm H_{, 0}+1} q^{-d_{1}}\left(v \otimes e^{\beta} e^{\Lambda P}\right) \\
& =q^{\mp\left(\alpha, \mid \beta+\Lambda_{p}\right)-1} z^{ \pm\left\langle h_{j}, \beta+\Lambda_{p}\right\rangle+1} \kappa^{\frac{1}{2} \sum_{k=0}^{n}\left\langle h_{1}, m_{k} \alpha_{k}\right\rangle m_{1 h}} v \otimes e^{ \pm \alpha \alpha_{j}} e^{\beta} e^{\Lambda_{p}} \\
& =\left(q^{-1} z\right)^{ \pm\left\langle h, \beta+\Lambda_{p}\right\rangle+1} \kappa^{\frac{1}{2} \sum_{k=0}^{n}\left\langle h_{,}, m_{k} \alpha_{k}\right\rangle m_{k} k} v \otimes e^{ \pm \alpha_{j}} e^{\beta} e^{\Lambda_{p}} \\
& =e^{ \pm \alpha}\left(q^{-1} z\right)^{ \pm H_{, 0}+1}\left(v \otimes e^{\beta} e^{\Lambda_{p}}\right) \text {. }
\end{aligned}
$$

Therefore we have

$$
\begin{aligned}
q^{d_{1}} E_{\jmath}(z) q^{-d_{1}} & =q^{d_{1}} \exp \left(\sum_{k \geq 1} \frac{H_{,-k}}{[k]} q^{-\frac{1}{2} k} z^{k}\right) \exp \left(-\sum_{k \geq 1} \frac{H_{3, k}}{[k]} q^{-\frac{1}{2}} z^{-k}\right) e^{\alpha_{\jmath}} z^{H_{, 0}+1} q^{-d_{1}} \\
& =\exp \left(\sum_{k \geq 1} \frac{H_{j,-k}}{[k]} q^{-\frac{1}{2} k}\left(q^{-1} z\right)^{k}\right) \exp \left(-\sum_{k \geq 1} \frac{H_{\jmath, k}}{[k]} q^{-\frac{1}{2} k}\left(q^{-1} z\right)^{-k}\right) e^{\alpha_{\jmath}}\left(q^{-1} z\right)^{H_{, 0}+1}
\end{aligned}
$$




$$
=E_{j}\left(q^{-1} z\right) .
$$

Similarly we have $q^{d_{1}} F_{j}(z) q^{-d_{1}}=F,\left(q^{-1} z\right)$.

It is clear that

$$
q^{d_{2}} e^{ \pm \alpha_{j}} z^{ \pm H_{j, 0}+1} q^{-d_{2}}\left(v \otimes e^{\beta} e^{\Lambda_{p}}\right)=q^{ \pm \delta_{j}} e^{ \pm \alpha_{1}} z^{ \pm H_{j, 0}+1}\left(v \otimes e^{\beta} e^{\Lambda_{p}}\right)
$$

From (A.1.13) and the fact that $q^{d_{2}}$ commutes with $H_{j, k}$, we have (A.1.8). We shall show (A.1.9). We denote

$$
\begin{aligned}
& E_{\imath}^{+}(z)=\exp \left(\sum_{k \geq 1} \frac{H_{l,-k}}{\left.[k]^{-\frac{1}{2} k} z^{k}\right),}\right. \\
& E_{i}^{-}(z)=\exp \left(-\sum_{k \geq 1} \frac{H_{i, k}}{\left.[k]^{-\frac{1}{2} k} z^{-k}\right) .}\right. \\
& F_{\imath}^{+}(z)=\exp \left(-\sum_{k \geq 1} \frac{H_{\imath,-k}}{[k]} q^{\frac{1}{2} k} z^{k}\right), \\
& F_{\imath}^{-}(z)=\exp \left(\sum_{k \geq 1} \frac{H_{1, k}}{\left.[k]^{\frac{1}{2} k} w^{-k}\right) .}\right.
\end{aligned}
$$

Let us proof

$$
K_{1}^{+}(z) E_{\jmath}(w)=\theta_{-\left\langle h_{r}, \alpha,\right\rangle}\left(q^{-\frac{1}{2}} \kappa^{m_{-i t}} \frac{w}{z}\right) E_{j}(w) K_{\imath}^{+}(z)
$$

We have

$$
\begin{aligned}
& {\left[\left(q-q^{-1}\right) \sum_{k \geq 1} H_{i . k} z^{-k}, \sum_{l \geq 1} \frac{H_{j .-l}}{[l]} q^{-\frac{1}{2} l} w^{l}\right]=\sum_{k, l}\left(q-q^{-1}\right) \frac{1}{[l]}\left[H_{l . k}, H_{, .-l}\right] z^{-k} q^{-\frac{1}{2} l} w^{l}} \\
& =\sum_{k}\left(q-q^{-1}\right) \frac{\left[k\left\langle h_{\imath}, \alpha_{j}\right\rangle\right]}{k} \kappa^{-k m_{v}} q^{-\frac{1}{2} k}\left(\frac{w}{z}\right)^{k} \\
& =\sum_{k} \frac{1}{k}\left(q^{k\left(\left\langle h_{t}, \alpha,\right\rangle-\frac{1}{2}\right)}-q^{k\left(-\left\langle h_{t}, \alpha,\right\rangle-\frac{1}{2}\right)}\right) \kappa^{-k m_{t s}}\left(\frac{w}{z}\right)^{k} \\
& =\log \frac{1-q^{-\left\langle h_{t}, \alpha_{\jmath}\right\rangle-\frac{1}{2}} \kappa^{-m_{t}} \frac{w}{z}}{1-q^{\left\langle h_{t}, \alpha_{\jmath}\right\rangle-\frac{1}{2}} \kappa^{-m_{t}} \frac{w}{z}}
\end{aligned}
$$

and

$$
\left[\left(q-q^{-1}\right) \sum_{k \geq 1} H_{\imath . k} z^{-k},-\sum_{l \geq 1} \frac{H_{j . l}}{[l]} q^{-\frac{1}{2} l} w^{-l}\right]=0
$$

We recall Campbell-Hausdorff formula: let $A$ and $B$ be noncommutative operators and $C=[A, B]$. If $[C, A]=[C, B]=0$ then we have $e^{A} e^{B}=e^{C} e^{B} e^{A}$.

By Campbell-Hausdorff formula we get 


$$
\begin{aligned}
& \exp \left(\left(q-q^{-1}\right) \sum_{k \geq 1} H_{i . k} z^{-k}\right) E_{j}^{+}(w) E_{j}^{-}(w) \\
& =\frac{1-q^{-\left\langle h_{t}, \alpha,\right\rangle-\frac{1}{2}} \kappa^{-m_{t}} \frac{w}{z}}{1-q^{\left\langle h_{1}, \alpha,\right\rangle-\frac{1}{2}} \kappa^{-m_{i}}, \frac{w}{z}} E_{J}^{+}(w) E_{\jmath}^{-}(w) \exp \left(\left(q-q^{-1}\right) \sum_{k \geq 1} H_{\imath . k} z^{-k}\right) .
\end{aligned}
$$

On the other hand, by Lemma 3.4.1 we have

$$
q^{\partial_{\alpha_{1}}} e^{\alpha_{3}} w^{H_{j, 0}}=q^{\left\langle h_{t}, \alpha\right\rangle} e^{\alpha^{\prime}} w^{H j, 0} q^{\partial_{\alpha_{1}}}
$$

Thus we get

$$
\begin{aligned}
& K_{i}^{+}(z) E_{j}(w) \\
& =\exp \left(\left(q-q^{-1}\right) \sum_{k \geq 1} H_{t, k} z^{-k}\right) E_{j}^{+}(w) E_{\jmath}^{-}(w) q^{\partial_{\alpha_{t}}} e^{\alpha_{\jmath}} w^{H_{\jmath, 0}+1} \\
& =\frac{1-q^{-\left\langle h_{t}, \alpha \jmath\right\rangle-\frac{1}{2}} \kappa^{-m_{t}} \frac{w}{z}}{1-q^{-\left\langle h_{t}, \alpha,\right\rangle-\frac{1}{2}} \kappa^{-m_{t}} \frac{w}{z}} E_{j}(w) K_{i}^{+}(z) \\
& =\theta_{-\left\langle h_{t}, \alpha,\right\rangle}\left(q^{-\frac{1}{2}} \kappa^{m_{-t}, \frac{w}{z}}\right) E_{\jmath}(w) K_{1}^{+}(z) .
\end{aligned}
$$

The other formulas in (A.1.9) can be checked by similar arguments.

Let us show (A.1.10). We have

$$
\begin{aligned}
& {\left[-\sum_{k \geq 1} \frac{H_{1, k}}{[k]} q^{-\frac{1}{2} k} z^{-k},-\sum_{k \geq 1} \frac{H_{j,-k}}{[k]} q^{\frac{1}{2} k} w^{k}\right]} \\
& =\sum_{k \geq 1} \frac{1}{k[k]}\left[k\left\langle h_{1}, \alpha_{\jmath}\right\rangle\right] \kappa^{-m_{t \prime}}\left(\frac{w}{z}\right)^{k}
\end{aligned}
$$

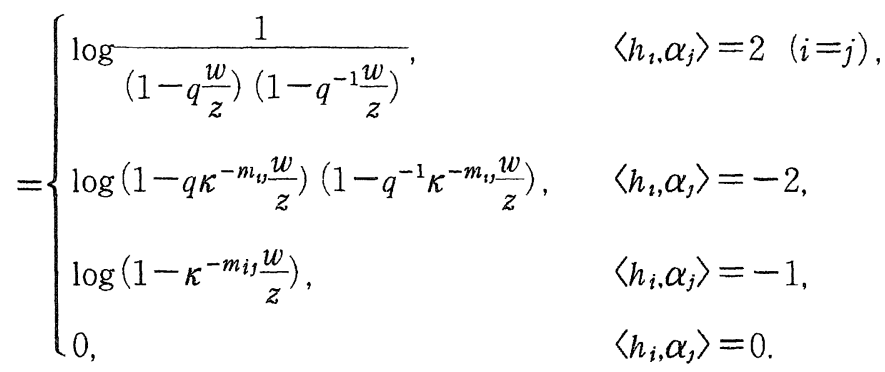

For example we will show in the case of $\left\langle h_{\imath}, \alpha_{j}\right\rangle=-2$. By CampbellHausdorff formula we get

and

$$
E_{\imath}^{ \pm}(z) F_{j}^{\ddagger}(w)=F_{j}^{ \pm}(w) E_{\imath}^{ \pm}(z)
$$




$$
E_{\imath}^{-}(z) F_{j}^{+}(w)=\left(1-q \kappa^{-m_{\imath}, \frac{w}{z}}\right)\left(1-q^{-1} \kappa^{-m_{\mathfrak{t}}} \frac{w}{z}\right) F_{j}^{+}(w) E_{\imath}^{-}(z)
$$

On the other hand, by Lemma 3.4.1, we have

$$
z^{H_{t, 0}} e^{-\alpha_{\jmath}}=z^{2} \kappa^{m_{i j}} e^{-\alpha_{s}} z^{H_{t, 0}} .
$$

Therefore we get

$$
\begin{aligned}
& E_{i}(z) F_{j}(w)=E_{\imath}^{+}(z) E_{\imath}^{-}(z) e^{\alpha_{\imath}} z^{H_{i, 0}+1} F_{j}^{+}(w) F_{\jmath}^{-}(w) e^{-\alpha_{\jmath}} w^{-H, 0^{+1}} \\
& =\left(z \kappa^{\frac{1}{2} m_{\imath}}-q w \kappa^{-\frac{1}{2} m_{\imath \jmath}}\right)\left(z \kappa^{\frac{1}{2} m_{\imath \jmath}}-q^{-1} w \kappa^{-\frac{1}{2} m_{\imath j}}\right) E_{\imath}^{+}(z) F_{j}^{+}(w) E_{\imath}^{-}(z) F_{j}^{-}(w) \\
& \times e^{\alpha_{j}} e^{-\alpha_{s}} z^{H_{t, 0}+1} w^{-H_{t, 0}+1} .
\end{aligned}
$$

By a similar argument we have

$$
\begin{aligned}
& F_{j}(w) E_{\imath}(z)=\left(w \kappa^{-\frac{1}{2} m_{\imath}}-q z \kappa^{\left.\frac{1}{2} m_{\imath}\right)}\right)\left(u \kappa^{-\frac{1}{2} m_{\imath}}-q^{-1} z \kappa^{\frac{1}{2} m_{\imath}}\right) E_{\imath}^{+}(z) F_{j}^{+}(w) E_{\imath}^{-}(z) F_{j}^{-}\left(u^{\prime}\right) \\
& \times e^{\alpha_{t}} e^{-\alpha_{t}} z^{H_{t, 0}+1} w^{-H_{t, 0}+1} .
\end{aligned}
$$

Therefore we get

$$
\left[E_{\imath}(z), F,(w)\right]=0
$$

Similarly one can check the other formulas.

We will show (A.1.11). We have

$$
\begin{aligned}
& {\left[-\sum_{k \geq 1} \frac{H_{i, k}}{[k]} q^{-\frac{1}{2} k} z^{-k}, \sum_{k \geq 1} \frac{H_{\lambda_{1}-k}}{[k]} q^{-\frac{1}{2} k} u^{k}\right]} \\
& =\sum_{k \geq 1} \frac{1}{k[k]}\left[k\left\langle h_{\imath}, \alpha_{j}\right\rangle\right] \kappa^{-m_{\imath},\left(\frac{w}{z}\right) k} \\
& = \begin{cases}\log \left(1-\frac{w}{z}\right)\left(1-q^{-2} \frac{w}{z}\right), & i=j, \\
\log \frac{1}{\left(1-\kappa^{-m_{\imath},} \frac{w}{z}\right)\left(1-q^{-2} \kappa^{-n_{\imath}, \frac{w}{z}}\right)}, & \left\langle h_{\imath}, \alpha_{\jmath}\right\rangle=-2, \\
\log \frac{1}{1-q^{-1} \kappa^{-n_{\imath}, \frac{w}{z}},} & \left\langle h_{\imath}, \alpha_{j}\right\rangle=-1, \\
0, & \left\langle h_{\imath}, \alpha_{\jmath}\right\rangle=0 .\end{cases}
\end{aligned}
$$

For example let us show in the case of $\left\langle h_{1}, \alpha_{j}\right\rangle=-2$. If $\left\langle h_{1}, \alpha_{j}\right\rangle \neq-2$ one can show the formula by a similar argument. By Lemma 3.4 .1 we get

$$
\left(z \kappa^{m_{\imath}}-q^{\left\langle h_{\imath}, \alpha,\right\rangle} w\right) E_{\imath}(z) E_{\jmath}(w)
$$




$$
\begin{aligned}
& =\left(z \kappa^{m_{\imath}}-q^{-2} w\right) E_{i}^{+}(z) E_{i}^{-}(z) e^{\alpha_{i}} z^{H_{s, 0+1}} E_{j}^{+}(w) E_{j}^{-}(w) e^{\alpha j} w^{H, 0+1} \\
& =\frac{z \kappa^{m_{1}}-q^{-2} w}{z^{2} \kappa^{m_{v}}\left(1-\kappa^{-m_{t}}, \frac{w}{z}\right)\left(1-q^{-2} \kappa^{-m_{t}} \frac{w}{z}\right)} E_{1}^{+}(z) E_{i}^{+}(w) E_{i}^{-}(z) E_{\jmath}^{-}(w) \\
& \times e^{\alpha_{t}} e^{\alpha \prime} z^{H_{t, 0+1}} w^{H ;, 0+1} \\
& =\frac{1}{\left(z-\kappa^{-m_{w} w} w\right)} E_{\imath}^{+}(z) E_{\imath}^{+}(w) E_{j}^{-}(z) E_{j}^{-}(w) e^{\alpha_{t}} e^{\alpha_{t}} z^{H_{t, 0+1}} u^{H, 0+1} .
\end{aligned}
$$

On the other hand we have

$$
\begin{aligned}
& \left(q^{\left\langle h_{i}, \alpha_{j}\right\rangle} \kappa^{m_{i j}} z-w\right) E_{j}\left(w^{\prime}\right) E_{\imath}(z)
\end{aligned}
$$

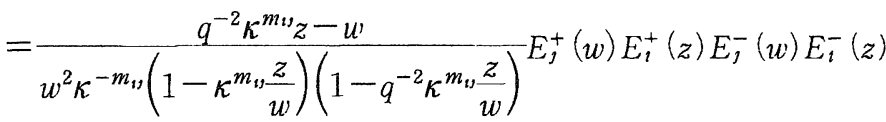

$$
\begin{aligned}
& \times e^{\alpha_{i}} e^{\alpha}, u^{H, 0+1} z^{H_{t, 0+1}} \\
& =\frac{1}{\left(z-\kappa^{-m t s} w\right)} E_{\imath}^{+}(z) E_{\jmath}^{+}(w) E_{\imath}^{-}(z) E_{j}^{-}(w) e^{\alpha_{t}} e^{\alpha_{\imath}} z^{H_{t, 0}+1} w^{H_{t} 0+1} \text {. }
\end{aligned}
$$

Thus we have $\left(z \kappa^{m_{t}}-q^{\left\langle h_{t}, \alpha \jmath\right\rangle} w\right) E_{\imath}(z) E_{\jmath}(w)=\left(q^{\left\langle h_{t}, \alpha_{\jmath}\right\rangle} \kappa^{m_{t v}} z-w\right) E,(w) E_{i}(z)$.

The formula $\left(z \kappa^{m_{\imath \jmath}}-q^{-\left\langle h_{t}, \alpha\right\rangle} w\right) F_{\imath}(z) F_{j}(w)=\left(q^{-\left\langle h_{t}, \alpha\right\rangle} \kappa^{m_{t} z} z-w\right) F_{j}(w) F_{1}(z)$ is proved similarly.

Let us prove (A.1.12). Assume that $\left\langle h_{\imath}, \alpha_{j}\right\rangle=-2$. This is the most complicated case. The other cases can be proved similarly.

We have following formulas:

$$
\begin{aligned}
& E_{\imath}\left(z_{1}\right) E_{\imath}\left(z_{2}\right) E_{l}\left(z_{3}\right) E_{j}(w)
\end{aligned}
$$

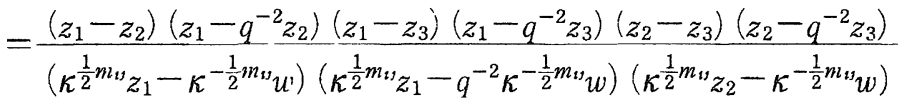

$$
\begin{aligned}
& \times \frac{1}{\left(\kappa^{\frac{1}{2} m w} z_{2}-q^{-2} \kappa^{-\frac{1}{2} m v} w\right)\left(\kappa^{\frac{1}{2} m u s} z_{3}-\kappa^{-\frac{1}{2} m u v} w\right)\left(\kappa^{\frac{1}{2} m u v} z_{3}-q^{-2} \kappa^{-\frac{1}{2} m u s} w\right)} \\
& \times E_{\imath}^{+}\left(z_{1}\right) E_{\imath}^{+}\left(z_{2}\right) E_{\imath}^{+}\left(z_{3}\right) E_{j}^{+}(w) E_{\imath}^{-}\left(z_{1}\right) E_{1}^{-}\left(z_{2}\right) E_{1}^{-}\left(z_{3}\right) E_{j}^{-}(w) \\
& \times e^{3 \alpha_{i}} e^{\alpha_{3}} z_{1}^{H_{t, 0+1}} z_{2}^{H_{t, 0+1}} z_{3}^{H_{t, 0+1}} w^{H_{, 0+1}}, \\
& E_{\imath}\left(z_{1}\right) E_{i}\left(z_{2}\right) E_{J}(w) E_{\imath}\left(z_{3}\right) \\
& =\frac{\left(z_{1}-z_{2}\right)\left(z_{1}-q^{-2} z_{2}\right)\left(z_{1}-z_{3}\right)\left(z_{1}-q^{-2} z_{3}\right)\left(z_{2}-z_{3}\right)\left(z_{2}-q^{-2} z_{3}\right)}{\left(\kappa^{\frac{1}{2} m_{v}} z_{1}-\kappa^{-\frac{1}{2} m_{w}} w\right)\left(\kappa^{\frac{1}{2} m_{v s}} z_{1}-q^{-2} \kappa^{-\frac{1}{2} m_{v}} w\right)\left(\kappa^{\frac{1}{2} m_{v}} z_{2}-\kappa^{-\frac{1}{2} m_{v}} w\right)}
\end{aligned}
$$


176

YoSHIHISA SAITO

$$
\begin{aligned}
& \times \frac{1}{\left(\kappa^{\frac{1}{2} m_{v}} z_{2}-q^{-2} \kappa^{-\frac{1}{2} m_{t}} w\right)\left(\kappa^{-\frac{1}{2} m_{v} w}-\kappa^{\frac{1}{2} m_{v} v} z_{3}\right)\left(\kappa^{-\frac{1}{2} m_{w} w}-q^{-2} \kappa^{\frac{1}{2} m u s} z_{3}\right)} \\
& \times E_{i}^{+}\left(z_{1}\right) E_{i}^{+}\left(z_{2}\right) E_{i}^{+}\left(z_{3}\right) E_{j}^{+}(w) E_{i}^{-}\left(z_{1}\right) E_{i}^{-}\left(z_{2}\right) E_{i}^{-}\left(z_{3}\right) E_{j}^{-}(w) \\
& \times e^{3 \alpha_{i}} e^{\alpha,} z_{1}^{H_{t, 0+1}} z_{2}^{H_{t, 0+1}} z_{3}^{H_{t, 0+1}} w^{H, 0+1} \text {, } \\
& E_{i}\left(z_{1}\right) E_{\jmath}(w) E_{\imath}\left(z_{2}\right) E_{\imath}\left(z_{3}\right) \\
& =\frac{\left(z_{1}-z_{2}\right)\left(z_{1}-q^{-2} z_{2}\right)\left(z_{1}-z_{3}\right)\left(z_{1}-q^{-2} z_{3}\right)\left(z_{2}-z_{3}\right)\left(z_{2}-q^{-2} z_{3}\right)}{\left(\kappa^{\frac{1}{2} m_{w}} z_{1}-\kappa^{-\frac{1}{2} m_{w}} w\right)\left(\kappa^{\frac{1}{2} m_{w}} z_{1}-q^{-2} \kappa^{-\frac{1}{2} m_{w} w} w\right)\left(\kappa^{-\frac{1}{2} m_{w} w}-\kappa^{\frac{1}{2} m_{v}} z_{2}\right)}
\end{aligned}
$$

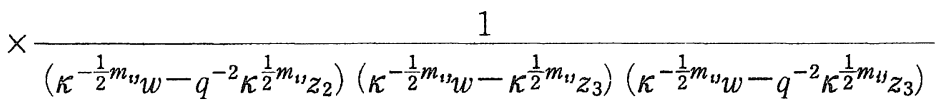

$$
\begin{aligned}
& \times E_{\imath}^{+}\left(z_{1}\right) E_{\imath}^{+}\left(z_{2}\right) E_{\imath}^{+}\left(z_{3}\right) E_{j}^{+}(w) E_{\imath}^{-}\left(z_{1}\right) E_{i}^{-}\left(z_{2}\right) E_{i}^{-}\left(z_{3}\right) E_{\jmath}^{-}(w) \\
& \times e^{3 \alpha_{i}} e^{\alpha_{s}} z_{1}^{H_{t, 0+1}} z_{2}^{H_{t, 0+1}} z_{3}^{H_{t, 0+1}} w^{H, 0+1}, \\
& E_{j}(w) E_{\imath}\left(z_{1}\right) E_{\imath}\left(z_{2}\right) E_{\imath}\left(z_{3}\right) \\
& =\frac{\left(z_{1}-z_{2}\right)\left(z_{1}-q^{-2} z_{2}\right)\left(z_{1}-z_{3}\right)\left(z_{1}-q^{-2} z_{3}\right)\left(z_{2}-z_{3}\right)\left(z_{2}-q^{-2} z_{3}\right)}{\left(\kappa^{-\frac{1}{2} m_{t}} w-\kappa^{\frac{1}{2} m_{t}} z_{1}\right)\left(\kappa^{-\frac{1}{2} m_{t} w} w-q^{-2} \kappa^{\frac{1}{2} m_{t}} z_{1}\right)\left(\kappa^{-\frac{1}{2} m_{\mathfrak{w}} w}-\kappa^{\frac{1}{2} m_{\mathfrak{t}}} z_{2}\right)}
\end{aligned}
$$

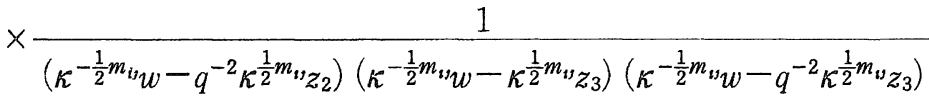

$$
\begin{aligned}
& \times E_{i}^{+}\left(z_{1}\right) E_{\imath}^{+}\left(z_{2}\right) E_{\imath}^{+}\left(z_{3}\right) E_{j}^{+}(w) E_{i}^{-}\left(z_{1}\right) E_{\imath}^{-}\left(z_{2}\right) E_{i}^{-}\left(z_{3}\right) E_{j}^{-}(w) \\
& \times e^{3 \alpha_{i}} e^{\alpha,} z_{1}^{H_{t, 0+1}} z_{2}^{H_{i, 0+1}} z_{3}^{H_{i, 0+1}} w^{H, 0+1} \text {. }
\end{aligned}
$$

Therefore it is enough to show that

$$
\begin{aligned}
& \sum_{\sigma \in \mathbb{E}_{3}} \frac{\prod_{i<j}\left(z_{\sigma(i)}-z_{\sigma(j)}\right)\left(z_{\sigma(i)}-q^{-2} z_{\sigma(j)}\right)}{\prod_{l=1}\left(\kappa^{\frac{1}{2} m_{s j}} z_{\sigma(\imath)}-\kappa^{\left.-\frac{1}{2} m_{v} w\right)}\right.}
\end{aligned}
$$

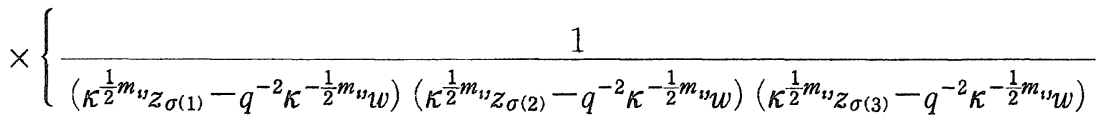

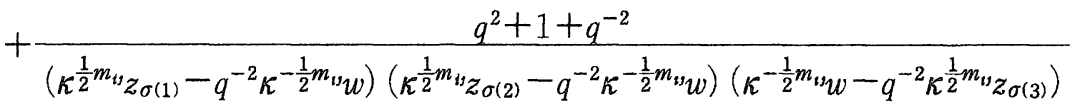

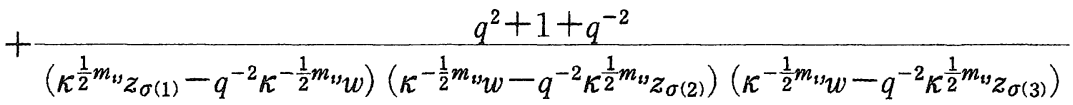




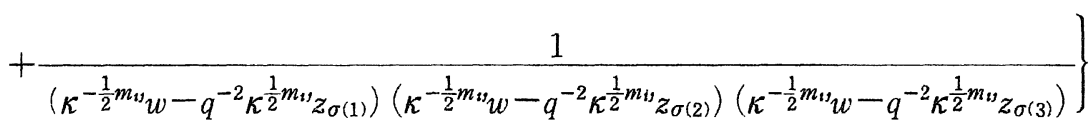

$=0$.

This identity is proved by a direct calculation.

Thus the proposition is proved.

\section{Acknowledgment}

The auther would like to thank Masaki Kashiwara. Tetsuji Miwa and Michio Jimbo for their encouragement and valuable discussions. He also thanks Kenji Iohara, Norio Suzuki, Eric Vasserot and Hiroshi Yamada for stimulating discussions.

\section{References}

[Be] Beck, J., Braid group action and quantum affine algebras, Comm. Math. Phys., 165 (1994), 555-568.

[B] Berman, S., On generators and relations for certain involutory subalgebras Kac-Moody Lie algebras. Comm. Alg., 17 (12) (1989) 3165-3185.

[BM] Berman, S. and Moody, R., Lie algebras graded by finite root systems and the intersection matrix algebras of Slodowy, Inv. Math., 108(1992), 323-347.

[C] Cherednik, I., Double-affine Hecke algebras, Knızhink-Zamolodchikov equatıons and Macdonald's operators, Intem. Math. Research Notices, 9(1992), 171-180.

[CP] Char1. V. and Pressly. A., Quantum affine algebras and affine Hecke algebras, Pacific J. Math., 174 (1996) , 295-326.

[D1] Drinfeld. V., Quantum groups, Proc. ICM Berkeley, 1 (1986), 789-820.

[D2] - A new realızation of Yangıans and quantized affıne algebras. Sov. Math. Dokl., 36 (1988), 212-216.

[EM] Eswara Rao, S. and Moody, R. V.. Vertex representations for $N$-toroidal Lie algebras and a Generalization of the Virasoro algebra, Comm. Math. Phys., 159 (1994), 239-264.

[FJ] Frenkel, I. and Jing, N., Vertex representations of quantum affine algebras, Proc. Natl. Acad. Scr. USA, 85 (1988), 9373-9377.

[FK] Frenkel, I. and Kac. V., Basic Representations of affine Lie algebras and dual Resonance models. Inv. Math., 62 (1980), 23-66.

[GKV] Ginzburg, V., Kapranov, M. and Vasserot, E., Langlands reciprocity for algebraic surface. Math. Res. Lett., 2(1995), 147-160.

[K] Koyama, Y., Staggered polarization of vertex model with $U_{q}(\hat{\underline{G}} \mid(n))$-symmetry, Comm. Math. Phys., 164 (1994), 277-291.

[MEY] Moody. R. V., Eswara Rao, S. and Yokomuma, T., Toroidal Lie algebras and vertex representations, Geom. Dedicata, 35(1990), 283-307.

[S] Slodowy. S., Bevond Kac-Moody algebras, and inside, Canadian Math. Soc. Conference Proccedings, 5 (1986), 361-371.

[V] Vasserot, E.. Private communication.

[VV] Varagnolo, M. and Vesserot, E., Schur duality in the toroidal setting, Comm. Math. Phys., 182 (1996), 469-484.

[Y] Yamada, H., Extended affine Lie algebra and their vertex representations, Publ. RIMS. Kyoto Univ.. 25 (1989), 587-603. 
